\title{
A multiscale force-based curved beam element for masonry arches
}

\author{
Paolo Di Re ${ }^{a, *}$, Daniela Addessi ${ }^{a}$, Elio Sacco ${ }^{b}$ \\ ${ }^{a}$ Department of Structural and Geotechnical Engineering, Sapienza University of Rome, \\ Via Eudossiana 18, 00184 Rome, Italy, \{paolo.dire, daniela.addessi\}@uniroma1.it \\ ${ }^{b}$ Department of Structures for Engineering and Architecture, University of Naples Federico II, \\ Via Claudio 21, 80125 Naples, Italy, elio.sacco@unina.it
}

\begin{abstract}
This paper presents a Timoshenko beam finite element for nonlinear analysis of planar masonry arches. Considering small displacement and strain assumption, the element governing equations are defined according to a force-based formulation that adopts three different parametrizations of the axis planar curve, permitting the exact description of the element geometry for arbitrarily curved arches. Specific quadrature techniques are illustrated to perform numerical integration over the curved axis. A two-scale arch-to-beam homogenization procedure reproduces the nonlinear response of periodic masonry materials, where an equivalent Timoshenko straight beam describes the behavior of the reference Unit Cell made of a single linear elastic brick and a nonlinear mortar layer. Formation of the hinges characterizing the collapse mechanism of the arch is detected taking advantage of the quadrature rule along the axis and a fracture energy based regularization technique is employed to avoid damage localization.

The proposed curved beam model is implemented in a standard FE analysis code and is used to perform several numerical applications. After validating the proposed formulation through benchmarking tests under linear elastic material response, the numerical simulation of four experimental

\footnotetext{
*Corresponding author: P. Di Re (paolo.dire@uniroma1.it)
} 
tests is shown, concerning circular and parabolic masonry arches undergoing in-plane bending. The numerical results are validated through experimental outcomes and micromechanical FE models.

Keywords: Masonry arch, multiscale model, damage, curved beam, force-based element.

\section{Introduction}

Until first decades of 1900, masonry has been the most used building technology in Europe for both civil buildings and cultural heritage constructions. However, due to low tensile strength of mortar and weak bond at interfaces with bricks and/or blocks, masonry structural elements exhibit a poor tensile/flexural resistance, that reduces the structure capacity and limits the design solutions [1]. For vertical dead loads, a widely adopted solution has long been the introduction of arches and vaults [2], as their particular geometry reduces presence of tensile stresses and increases carrying capacity of the structure (shape-based resistance). Arches are especially used in wall panels and for long span bridges, as their behavior under dead loads is easy to understand and predict. However, the response of curved elements under variable loads is complex and its simulation can be a hard task [3, 4], particularly for horizontally seismic actions. Damage and micro-cracks usually develop along arch axis, even for small load levels, mainly due to debonding phenomena at interfaces and mortar failure. These influence the overall nonlinear response of the structure and determine collapse mechanisms. Hence, the development of efficient numerical tools for nonlinear analysis of masonry arches still represents a relevant issue for assessment of safety and strengthening of existing structures.

Many different models have been proposed to reproduce response of masonry structural elements and, in particular, masonry arches, commonly classified as mechanism methods (e.g. limit analysis), discrete element methods, discontinuous deformation analysis and finite element (FE) models [5-7]. The latter result the most adopted and flexible option, as these permit a complete representation of the nonlinear mechanisms with a reasonable demand in terms of computational costs. Micromechanical, macromechanical and multiscale approaches are three widely employed alternatives for FE analyses of masonry [8]. The first are based on distinct modeling of brick/blocks, mortar joints and/or interfaces; the second model masonry as an equivalent homogenized material and adopt phenomenological constitutive laws; the latter evaluate the response of a Unit Cell (UC) at a lower geometric scale and derive the response of the equivalent homogeneous medium at macro-scale through a homogenization process [9]. Multiscale models are a good compromise between detailed geometric/mechanical representation of the material and computational efforts.

Focusing on masonry arches, beam FEs can be suitably used to model the curved elements 
and define multiscale procedures, as these provide very accurate results compared to the limited computational burden required. Among different beam FE models, last decades have witnessed a large spread of force-based (FB) and mixed formulations for numerical analyses. As opposed to classic displacement-based (DB) models, where cross-section displacements are interpolated along the element axis by means of shape functions, FB beam formulations, also known as flexibility approaches, interpolate internal forces by means of functions satisfying element equilibrium in a strict sense. Hence, the selected interpolation functions are the exact solution to equilibrium equations regardless the element geometry and material constitutive law $[10,11]$. This is a consolidated procedure for straight beams and has shown superior performances with respect to DB models in reproducing the structural response [12], particularly under nonlinear material behavior [13, 14]. In fact, accurate inelastic solutions can be obtained significantly reducing the number of the elements used for the discretization. The FB beam formulation has also been proven to be free from shear locking, giving the exact analytical response for the elastic Timoshenko beam theory with a single FE per span $[15,16]$. A number of alternative strategies have been proposed to overcome the shear locking affecting the classic displacement-based FE formulations, such as selective reduced integration, use of quadratic polynomials transverse displacement and linear interpolation for the rotation, or interdependent interpolation of the transverse deflection and rotation derived on the basis of the exact solution for the Timoshenko beam theory [17]. Assumed strain methods have also been efficiently adopted, where the strains are independently interpolated and shear locking phenomena are avoided [18].

However, for both DB and FB formulations, few models consider the curved axis geometry, as the displacement and stress interpolation procedures are harder to define. The adoption of straight FEs combined with a mesh refinement is usually a preferred option to model the geometry of curved elements.

Besides the many proposals limited to linear elastic material assumptions and usually employed for large displacements and/or vibration analyses [19-24], the models in [25, 26] are worth to be mentioned for nonlinear material modeling, yet belonging to the DB formulations. For twodimensional (2D) arches, Molari and Ubertini [27] have proposed a robust and efficient FB beam model, where the curved axis geometry is approximated with a cubic interpolation scheme. This approach allows the analysis of arbitrarily curved elements and, thus, results in a general formulation for the analysis of planar curved structural members. However, the study in [27] is limited to linear elastic material responses and does not investigate the model capabilities in the nonlinear material range. Moreover, the interpolation procedure for the axis approximation requires a pre-processing phase, where geometric parameters are determined for each structural members.

This work presents a general FB Timoshenko model for 2D curved beams under both linear 
elastic and nonlinear material responses, assuming small displacement and strain hypotheses. The element formulation considers the classic six displacement nodal degrees of freedom (DOF) and provides an exactly equilibrated representation of the cross-section stress resultants. In addition to the case of arbitrarily curved beams, this work considers two special cases for the axis geometry description, common in civil structures: constant curvature beams (circular arches) and parabolic arches. For both cases, the element governing equations can be easily derived relying on the exact representation of the axis geometry through a suitable parametrization of the $2 \mathrm{D}$ curve equation. This approach considerably simplifies model formulation and does not require any pre-processing procedure to describe the geometry of the structure. Moreover, specific quadrature techniques are proposed to efficiently evaluate integrals over the axis involved in the element governing equations. These depend on the chosen geometric parametrization and strongly influence the computational costs.

The multiscale model proposed in [28] is introduced for the evaluation of the mechanical response of the general element cross-section. At the micro-scale level, a one-dimensional (1D) beam model describes the behavior of the UC and the homogenized macroscopic stress resultants at the curved beam quadrature point associated to the UC are determined via a semi-analytical approach. The bricks are considered as elastic, while a damage-friction constitutive law is used for the mortar.

In this model, the nonlinear hinges forming along the arch coincide with the zones where damage and plasticity localize and consequently their formation is monitored by following the evolution of the nonlinear mechanisms at the quadrature cross-sections. As usual when considering softening materials, the damage localization requires the adoption of a regularization technique. The most common regularization approaches are the nonlocal integral procedures, the gradient methods [29], the fracture-energy based techniques [30] and the approaches proposed by Addessi and Ciampi [14] and Scott and Hamutçuoğlu [31] for FB beams. Nonlocal integral procedures and gradients methods are hard to introduce in the element formulation and increase the computational burden, whereas the generalization of the approach in [14] to curved beams is not straightforward. Hence, to prevent damage localization issues, a fracture energy regularization method is used in this work, where the evolutionary parameters of the damage model are calibrated at each cross-section considering the associated length defined for the quadrature rule.

The proposed curved beam model is implemented in a standard FE analysis code and used to perform various numerical applications. Two tests validate the model and the proposed quadrature techniques for linear elastic material response and investigate its advantages with respect to existing proposals. Nonlinear analyses on real masonry structures are also performed to study the capability of the model in detecting the nonlinear hinge formation and the collapse mechanism of the mem- 
bers. The numerical results are validated through experimental outcomes and micromechanical FE models.

\section{FE model for 2D shear-deformable arches}

This section describes the FB formulation of a 2D curved beam FE for the analysis of structural arches under nonlinear material response. The plane curve representing the element axis is assumed to have $C^{0}$ continuity and small displacement and strain assumptions hold. The FE model is based on the classic Timoshenko beam theory and results free from shear-locking problems.

The description focuses on the most suitable coordinate parametrization for the analytical representation of the axis geometry. First, the general case is described, where the $2 \mathrm{D}$ curve defining the beam axis is assumed to be known in a general parametric representation of the two coordinate functions. Then, the formulations for the circular and parabolic arches are presented. The derivation for the parabolic arch is introduced as an example procedure to obtain the element governing equations when the beam axis geometry is known in explicit form.

As usual in nonlinear beam FEs [14, 31], the integrals over the axis involved in the element governing equations are evaluated through numerical integration procedures. Hence, the following study also describes the numerical approaches applied for the different beam formulations. These depend on the specific parametrization adopted to describe the axis geometry and permit to extend the model in [27] to the case of material nonlinear responses.

\subsection{General framework}

Referring to the global reference system $(\widehat{X}, \widehat{Y})$, in the general undeformed configuration of the beam (Fig. 1(a)) each element cross-section is orthogonal to the curved axis delimited by nodes $I$ and $J$. Six DOFs govern the element kinematics at the local level, that is two translations and one in-plane rotation at each node, collected in the displacement vector $\mathbf{U}$ as follows:

$$
\mathbf{U}=\left(\begin{array}{llllll}
U_{X, I} & U_{Y, I} & \Theta_{I} & U_{X, J} & U_{Y, J} & \Theta_{J}
\end{array}\right)^{T}
$$

The corresponding nodal forces are collected in the force vector $\mathbf{P}$. The direction going from $I$ to $J$ defines axis $X$ of the local reference system $(X, Y)$. According to the equilibrated approach [13], element rigid body motions are eliminated and the formulation refers to the local basic reference system in Fig. 1(b). Here, only three nodal deformation displacements are considered: the element

elongation $\widetilde{U}_{J}$ along $X$ and the nodal rotations $\widetilde{\Theta}_{I / J}$, listed in the basic displacement vector $\mathbf{V}=$ 


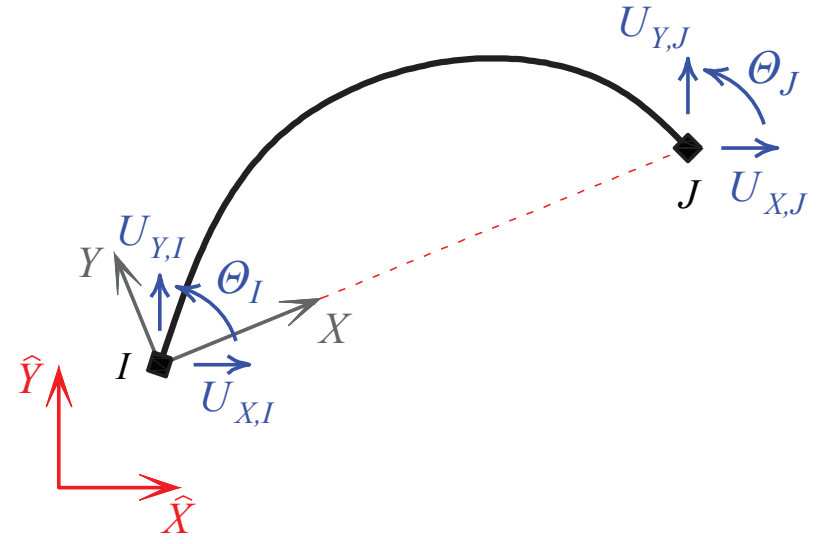

(a) Global reference system.

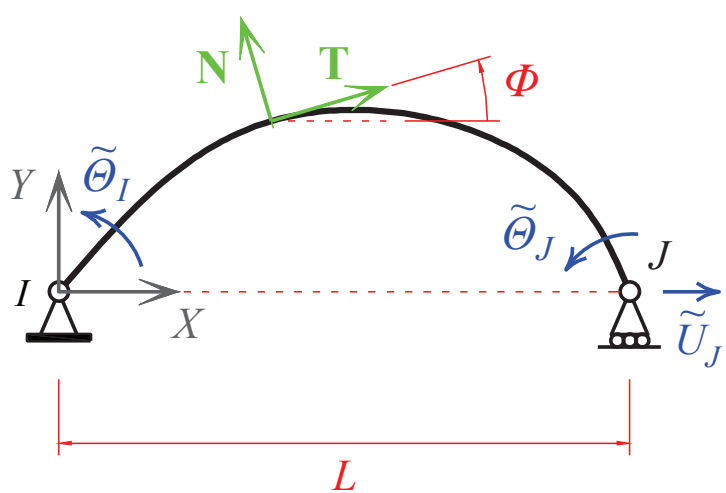

(b) Basic reference system.

Figure 1: Finite element local basic reference system and displacement vector components.

$\left(\begin{array}{lll}\widetilde{U}_{J} & \widetilde{\Theta}_{I} & \widetilde{\Theta}_{J}\end{array}\right)^{T}$. The kinematic relationship between $\mathbf{U}$ and $\mathbf{V}$ results as:

$$
\mathbf{V}=\mathbf{A}_{g} \mathbf{U}=\underbrace{\left[\begin{array}{cccccc}
-1 & 0 & 0 & 1 & 0 & 0 \\
0 & \frac{1}{L} & 1 & 0 & -\frac{1}{L} & 0 \\
0 & \frac{1}{L} & 0 & 0 & -\frac{1}{L} & 1
\end{array}\right]}_{\mathbf{A}_{v}} \mathbf{A}_{r} \mathbf{U}
$$

where matrix $\mathbf{A}_{g}=\mathbf{A}_{v} \mathbf{A}_{r}$ is the element compatibility matrix, depending on the undeformed element length $L$ and orientation. This rotates the nodal displacements from the global to local reference system, through the rotation matrix $\mathbf{A}_{r}$, and removes the element rigid body motions, through the kinematic matrix $\mathbf{A}_{v}$ [32]. At each point of the axis $\mathcal{L}$, unit vectors $\mathbf{T}$ and $\mathbf{N}$ are introduced representing tangent and normal directions to the curve. $\mathbf{T}$ forms the angle $\Phi$ with $X$ and $\mathbf{N}$ is assumed parallel to one of the cross-section central inertia axis.

The basic element force vector $\mathrm{Q}=\left(\begin{array}{lll}N_{J} & M_{I} & M_{J}\end{array}\right)^{T}$ contains the quantities work-conjugate to $\mathbf{V}$, where $N_{J}$ is the force parallel to $X$ at $J$ and $M_{I / J}$ is the in-plane nodal moment at $I / J$.

To enforce the equilibrium conditions that govern the force-based formulation, a convenient parametrization for the 2D curve defining the beam axis is required. Referring to the basic reference system, in general, different alternatives are available, commonly classified as: implicit representations, in the form $\mathcal{L} \equiv f(X, Y)=0$; explicit representations, in the form $\mathcal{L} \equiv Y=f(X)$; parametric representations, in the form $\mathcal{L} \equiv[X=f(\Lambda) ; Y=g(\Lambda)]$, with $\Lambda$ denoting a geometric variable. Adopting the parametric representation, Molari and Ubertini [27] have developed a gen- 


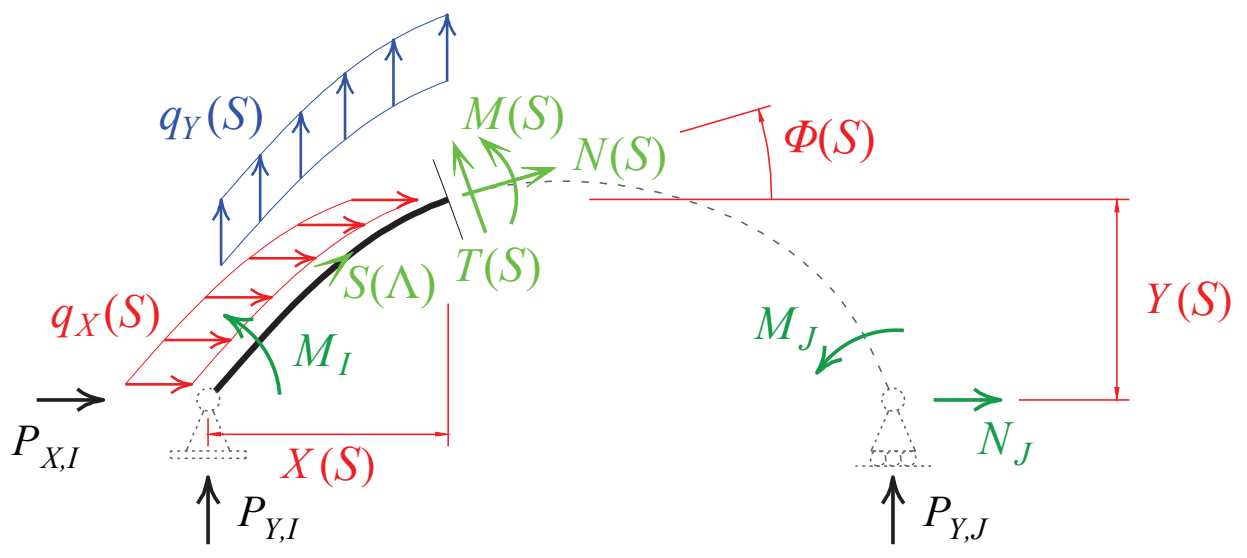

Figure 2: Equilibrated external and internal forces for the general portion of the element delimited by node $I$ and cross-section at $S(\Lambda)$.

eral FB formulation for arbitrarily curved arches. Indeed, when the coordinate functions $X(\Lambda)$ and $Y(\Lambda)$ are available in this form, the force-based FE formulation can be classically derived [10]. By contrast, if this is not the case, some alternative strategies can be adopted, consisting in (a) approximation of the coordinate functions $X(\Lambda)$ and $Y(\Lambda)$, e.g. using a cubic Hermite interpolation (as proposed by the same authors [27]) or spline interpolation and (b) adoption of different parametrizations.

Due to its wide applicability, formulation for the arbitrarily curved arch parametrized with respect to a general geometric variable is reviewed in the following section, referring to the general framework described above. The subsequent sections present the alternative (b) to derive the FE formulation with no geometric approximation in the case of circular and parabolic arches.

\subsection{FB formulation for arbitrarily curved arches}

The coordinate functions of the beam axis are assumed to be known in the parametric representation $[X(\Lambda) ; Y(\Lambda)]$, where $\Lambda$ ranges between $\Lambda_{0}$ and $\Lambda_{s}$ denoting values assumed by the geometric variable at the two ends of the curved axis. The arc-length abscissa $S$ (Fig. 2) is often adopted as geometric variable, ranging from 0 to the total element axis length $L_{s}$, although any parametrization can be used. A bijective relationship is assumed to exist between $\Lambda$ and $S$, that is each value of $\Lambda$ identifies one cross-section located at the curvilinear abscissa $S$ along $\mathcal{L}$, i.e. $S=S(\Lambda)$ and $\Lambda=\Lambda(S)$. The infinitesimal arch length is denoted as $d S$.

For a 2D Timoshenko beam, the generalized section stress vector $\Sigma(S)$ collects the three stress resultants, namely axial stress $N(S)$, bending moment $M(S)$ and shear stress $T(S)$. According 
to the FB formulation, the stress resultants that exactly satisfy the element equilibrium equations under small displacements are expressed by the following interpolatory relationship:

$$
\underbrace{\left(\begin{array}{c}
N(S) \\
M(S) \\
T(S)
\end{array}\right)}_{\boldsymbol{\Sigma}(S)}=\underbrace{\left[\begin{array}{ccc}
\cos \Phi(S) & -\frac{\sin \Phi(S)}{L} & -\frac{\sin \Phi(S)}{L} \\
Y(S) & \frac{X(S)}{L}-1 & \frac{X(S)}{L} \\
-\sin \Phi(S) & -\frac{\cos \Phi(S)}{L} & -\frac{\cos \Phi(S)}{L}
\end{array}\right]}_{\mathbf{B}(S)} \underbrace{\left(\begin{array}{c}
N_{J} \\
M_{I} \\
M_{J}
\end{array}\right)}_{\mathbf{Q}}+\underbrace{\left(\begin{array}{c}
N_{q}(S) \\
M_{q}(S) \\
T_{q}(S)
\end{array}\right)}_{\boldsymbol{\Sigma}_{q}(S)}
$$

In Eq. (3), $\mathbf{B}(S)$ is the equilibrium matrix, expressed in terms of angle $\Phi(S)$ and coordinates $X(S)$ and $Y(S)$ of the cross-section at $S$, whereas $\Sigma_{q}(S)$ contains the section stress resultants due to distributed loads. Fig. 2 shows a schematic representation of the variables involved in equilibrium equations. Here, $P_{X, I}, P_{Y, I}$ and $P_{Y, J}$ are the nodal reaction force components of $\mathbf{P}$ and consist of two contributions: one, $P_{X, I}^{b}, P_{Y, I}^{b}$ and $P_{Y, J}^{b}$, due to the basic forces $\mathbf{Q}$, the other, $P_{X, I}^{q}, P_{Y, I}^{q}$ and $P_{Y, J}^{q}$, due to the distributed loads. The latter are collected in the element nodal reaction force vector $\mathbf{P}_{q}$ due to $\mathbf{q}$, which assumes in the basic reference system the following:

$$
\mathbf{P}_{q}=\left(\begin{array}{llllll}
P_{X, I}^{q} & P_{Y, I}^{q} & 0 & 0 & P_{Y, J}^{q} & 0
\end{array}\right)^{T}
$$

With no loss of generality, the following description considers loads distributed along the element arc-length. The two load components $q_{X}(S)$ and $q_{Y}(S)$, parallel to $X$ and $Y$ respectively, are collected in the vector $\mathbf{q}=\left(\begin{array}{ll}q_{X} & q_{Y}\end{array}\right)^{T}$. For a general distributed load configuration, the following expressions hold:

$$
\begin{aligned}
N_{q}(S) & =-\left[P_{X, I}^{q}+Q_{X}(S)\right] \cos \Phi(S)-\left[P_{Y, I}^{q}+Q_{Y}(S)\right] \sin \Phi(S) \\
M_{q}(S) & =-P_{X, I}^{q} Y(S)+P_{Y, I}^{q} X(S)-M_{Y}(S)+M_{X}(S) \\
T_{q}(S) & =\quad\left[P_{X, I}^{q}+Q_{X}(S)\right] \sin \Phi(S)-\left[P_{Y, I}^{q}+Q_{Y}(S)\right] \cos \Phi(S)
\end{aligned}
$$

where:

$$
\begin{array}{llll}
Q_{X}(S) & =\int_{0}^{S} q_{X}(Z) d Z & & M_{Y}(S)=\int_{0}^{S} q_{X}(Z)[Y(S)-Y(Z)] d Z \\
Q_{Y}(S)=\int_{0}^{S} q_{Y}(Z) d Z & , & M_{X}(S)=\int_{0}^{S} q_{Y}(Z)[X(S)-X(Z)] d Z
\end{array}
$$

By enforcing the virtual work equivalence [33], that is by imposing that the nodal virtual work is equal to the internal virtual work of the generalized section stresses in equilibrium with $\mathrm{Q}$, under 
the assumption $\Sigma_{q}=0$, it results:

$$
\delta \mathbf{Q}^{T} \mathbf{V}=\int_{0}^{L_{s}} \delta \boldsymbol{\Sigma}^{T} \mathbf{E} d S
$$

where the generalized section strain vector $\mathbf{E}(S)$ work-conjugate to $\Sigma(S)$ collects axial elongation $E(S)$, curvature $K(S)$ and shear strain $\Gamma(S)$. Hence, by substituting Eq. (3) into Eq. (7) and removing $\delta \mathbf{Q}^{T}$ gives the standard compatibility conditions for the FB formulation:

$$
\mathbf{V}=\int_{0}^{L_{s}} \mathbf{B}^{T}(S) \mathbf{E}(S) d S
$$

The generalized constitutive law relating $\mathbf{E}(S)$ and $\Sigma(S)$ involves the consistent tangent generalized section flexibility matrix $\Upsilon(S)$ and results in incremental form as:

$$
\dot{\mathbf{E}}(S)=\Upsilon(S) \dot{\Sigma}(S)
$$

This depends on the specific constitutive model adopted to represent the material behavior (e.g. Sect. 3).

By differentiating Eq. (8) with respect to Q and introducing Eqs. (3) and (9), the consistent tangent element flexibility matrix $\mathbf{F}$ is obtained as:

$$
\mathbf{F}=\frac{\partial \mathbf{V}}{\partial \mathbf{Q}}=\int_{0}^{L_{s}} \mathbf{B}^{T}(S) \mathbf{\Upsilon}(S) \mathbf{B}(S) d S
$$

This governs the element basic constitutive relationship in the incremental form $\dot{\mathbf{Q}}=(\mathbf{F})^{-1} \dot{\mathbf{V}}$.

Following the procedure described in [33], the consistent tangent element stiffness matrix $\mathbf{K}$ and force vector $\mathbf{P}$ in the global reference system are obtained, related to the basic variables through matrix $\mathbf{A}_{g}$, i.e.:

$$
\mathbf{K}=\mathbf{A}_{g}^{T}(\mathbf{F})^{-1} \mathbf{A}_{g} \quad, \quad \mathbf{P}=\mathbf{A}_{g}^{T} \mathbf{Q}+\mathbf{A}_{r}^{T} \mathbf{P}_{q}
$$

Ihe integrals in Eqs. (6), (8) and (10) can be rewritten in terms of $\Lambda$, being the infinitesimal arch length equal to:

$$
d S=\left|\mathbf{X}^{\prime}(\Lambda)\right| d \Lambda=\sqrt{\left(\frac{\partial X(\Lambda)}{\partial \Lambda}\right)^{2}+\left(\frac{\partial Y(\Lambda)}{\partial \Lambda}\right)^{2}} d \Lambda
$$

The term $\left|\mathbf{X}^{\prime}(\Lambda)\right|$ transforms the infinitesimal length $d \Lambda$ into the corresponding $d S$. Then, to perform the numerical integration the geometric variable $\Lambda \in\left[\Lambda_{0} ; \Lambda_{S}\right]$ is linearly mapped into the 
rectilinear reference coordinate $\xi \in[-1 ;+1]$ and the following transformations [34] are used:

$$
\int_{0}^{L_{s}} f(S) d S=\int_{\Lambda_{0}}^{\Lambda_{S}} f(S(\Lambda))\left|\mathbf{X}^{\prime}(\Lambda)\right| d \Lambda=\frac{L_{s}}{2} \int_{-1}^{+1} \widetilde{f}(\xi)\left|\mathbf{X}^{\prime}(\xi)\right| d \xi
$$

where $\tilde{f}(\xi)$ is function $f(S)$ expressed in terms of $\xi$, i.e. $\widetilde{f}(\xi)=f \circ S \circ \Lambda$. The right-hand-side integral in Eq. (13) is finally solved through a standard quadrature scheme, where $n_{g}$ is the total number of quadrature cross-sections and $\xi_{n}$ and $w_{n}$, with $n=1, \ldots, n_{g}$, are the quadrature point locations and weights in the reference interval $[-1 ;+1]$. The quantity $L_{n}=\frac{L_{s}}{2}\left|\mathbf{X}^{\prime}\left(\xi_{n}\right)\right| w_{n}$ is the quadrature length associated to the $n$-th cross-section.

\subsection{FB formulation for circular arches}

Referring to the case of beam axis with constant curvature $\rho=1 / R$, the element governing equations in Sect. 2.2 can be easily rewritten expressing the arc-length abscissa as a linear function of the angle $\Psi$ between section plane and axis $X$, assumed as geometric independent variable. Referring to Fig. 3, where $C$ indicates center of the arch and $\Psi_{0}$ abutment angle, the increment of the arc-length abscissa results as $d S=R d \Psi$ and the relationship between $S$ and $\Psi$ is written as $S(\Psi)=R\left(\Psi-\Psi_{0}\right)$, with $\Psi$ ranging from $\Psi_{0}$ to $\pi-\Psi_{0}$. Hence, considering that $\Phi(\Psi)=\pi / 2-\Psi$, the equilibrium conditions in Eq. (3) become:

$$
\underbrace{\left(\begin{array}{c}
N(\Psi) \\
M(\Psi) \\
T(\Psi)
\end{array}\right)}_{\boldsymbol{\Sigma}(\Psi)}=\underbrace{\left[\begin{array}{ccc}
\sin \Psi & -\frac{\cos \Psi}{L} & -\frac{\cos \Psi}{L} \\
Y(\Psi) & \frac{X(\Psi)}{L}-1 & \frac{X(\Psi)}{L} \\
-\cos \Psi & -\frac{\sin \Psi}{L} & -\frac{\sin \Psi}{L}
\end{array}\right]}_{\mathbf{B}(\Psi)} \underbrace{\left(\begin{array}{c}
N_{J} \\
M_{J} \\
M_{J}
\end{array}\right)}_{\mathbf{Q}}+\underbrace{\left(\begin{array}{c}
N_{q}(\Psi) \\
M_{q}(\Psi) \\
T_{q}(\Psi)
\end{array}\right)}_{\mathbf{\Sigma}_{q}(\Psi)}
$$

Similarly, all the integrals in Eqs. (6) to (10) are evaluated with respect to $\Psi$. For instance, element compatibility condition in Eq. (8) and expression for the element flexibility matrix in Eq. (10) become:

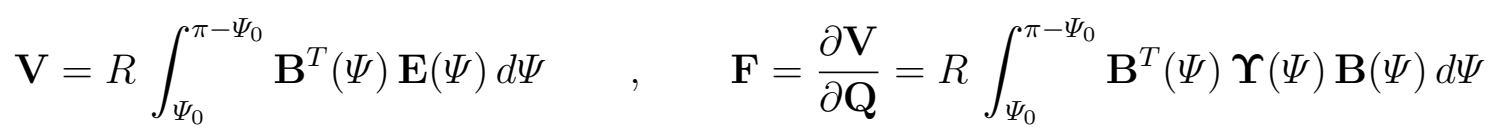

A special solution for the contribution due to distributed loads can be obtained when these are constant along the axis arc-length, that is $q_{X}(S)=\bar{q}_{X}$ and $q_{Y}(S)=\bar{q}_{Y}$. In this case, the nodal force components in Eq. (5) become:

$$
P_{X, I}^{q}=-\bar{q}_{X} L_{s} \quad, \quad P_{Y, I}^{q}=-\bar{q}_{Y} \frac{L_{s}}{2}-T_{X} \quad, \quad P_{Y, J}^{q}=-\bar{q}_{Y} \frac{L_{s}}{2}+T_{X}
$$




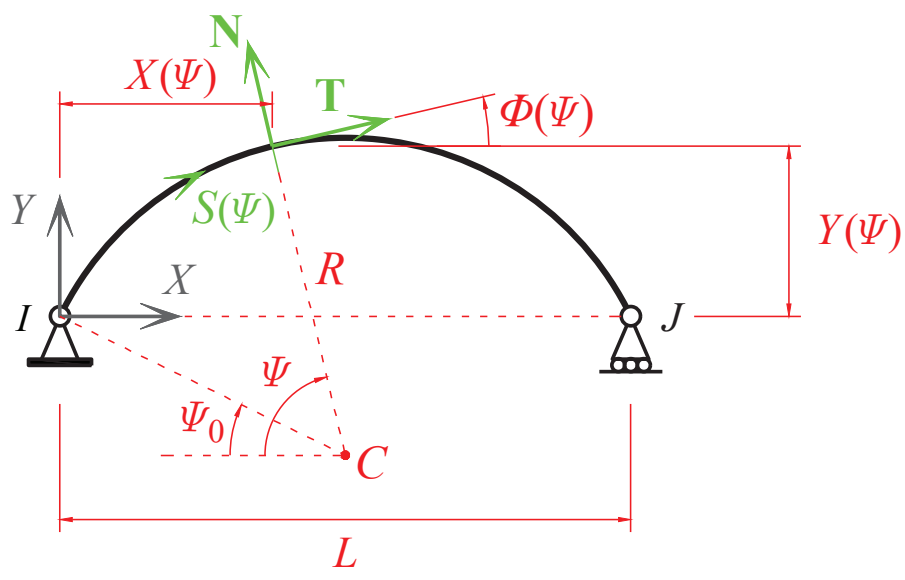

Figure 3: Geometric parametrization for the 2D curve representing the axis of the circular arch.

with $T_{X}=\frac{R^{2}}{L} \bar{q}_{X}\left[2 \cos \Psi_{0}+\sin \Psi_{0}\left(2 \Psi_{0}-\pi\right)\right]$ and Eq. (6) simplifies to:

$$
\begin{array}{lll}
Q_{X}(\Psi)=\bar{q}_{X} R\left(\Psi-\Psi_{0}\right) & , & M_{Y}(\Psi)=\bar{q}_{X} R^{2}\left[\cos \Psi-\cos \Psi_{0}-\sin \Psi\left(\Psi_{0}-\Psi\right)\right] \\
Q_{Y}(\Psi)=\bar{q}_{Y} R\left(\Psi-\Psi_{0}\right) & , & M_{X}(\Psi)=\bar{q}_{Y} R^{2}\left[\sin \Psi-\sin \Psi_{0}+\cos \Psi\left(\Psi_{0}-\Psi\right)\right]
\end{array}
$$

To numerically evaluate the integrals appearing in the element governing equations (e.g. Eqs. (15)), it is useful to consider the axis curve parametrization based on the angle $\Psi$. Indeed, to perform the numerical integration the geometric variable $\Psi \in\left[\Psi_{0} ; \pi-\Psi\right]$ is mapped into the reference coordinate $\xi \in[-1 ;+1]$ (Fig. 4$)$ and it results:

$$
\int_{0}^{L_{s}} f(S) d S=R \int_{\Psi_{0}}^{\pi-\Psi_{0}} \widehat{f}(\Psi) d \Psi=R \frac{\pi-2 \Psi_{0}}{2} \int_{-1}^{+1} \widetilde{f}(\xi) d \xi \simeq R \frac{\pi-2 \Psi_{0}}{2} \sum_{n=1}^{n_{g}} \widetilde{f}\left(\xi_{n}\right) w_{n}
$$

where $\widehat{f}(\Psi)=f \circ S$ and $\tilde{f}(\xi)=f \circ S \circ \Psi$. In this case, $\Psi_{n}=\frac{\pi-2 \Psi_{0}}{2}\left(1+\xi_{n}\right)+\Psi_{0}$ and $L_{n}=$ $R \frac{\pi-2 \Psi_{0}}{2} w_{n}$ are the angle coordinate and the quadrature length associated to the $n$-th cross-section, respectively, as the Jacobian of the transformation between $\Psi$ and $\xi$ results as $|J|=\frac{\pi-2 \Psi_{0}}{2}$.

\subsection{FB formulation for parabolic arches}

Focusing on masonry panels, parabolic arches are usually defined with the parabola symmetry axis parallel to the global vertical axis. For these, the explicit expressions of the coordinate functions in terms of the arc-length abscissa $S$ are not available. In this case, the derivation of the element governing equations in Sect. 2.2 can be performed for the exact geometry. Referring to the coordinate system $(\bar{X}, \bar{Y})$ parallel to the global one and with origin in $I$ (Fig. 5), the axis curve in the explicit 


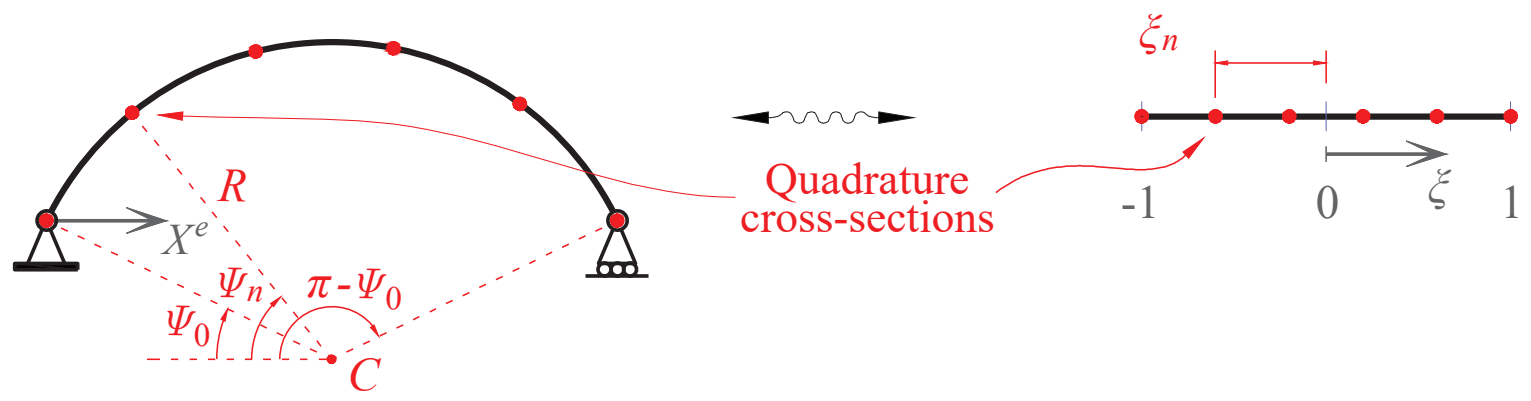

Figure 4: General numerical integration performed by mapping the geometric angle $\Psi \in\left[\Psi_{0} ; \pi-\Psi\right]$ into the reference coordinate $\xi \in[-1 ;+1]$.

form is expressed as:

$$
\bar{Y}=f(\bar{X})=a \bar{X}^{2}+b \bar{X}
$$

where $a$ and $b$ are coefficients evaluated on the basis of the particular axis geometry. Abscissa $\bar{X}$ is, thus, used as geometric independent variable, ranging from 0 to $\bar{L}=L \cos \Xi$ and angle $\Phi$ results as:

$$
\Phi(\bar{X})=\arctan \frac{\partial \bar{Y}(\bar{X})}{\partial \bar{X}}-\Xi=\arctan (2 a \bar{X}+b)-\Xi
$$

where $\Xi$ is the angle between $X$ and $\bar{X}$. Hence, the equilibrium conditions in Eq. (3) become:

$$
\underbrace{\left(\begin{array}{c}
N(\bar{X}) \\
M(\bar{X}) \\
T(\bar{X})
\end{array}\right)}_{\mathbf{\Sigma}(\bar{X})}=\underbrace{\left[\begin{array}{ccc}
\cos \Phi(\bar{X}) & -\frac{\sin \Phi(\bar{X})}{L} & -\frac{\sin \Phi(\bar{X})}{L} \\
Y(\bar{X}) & \frac{X(\bar{X})}{L}-1 & \frac{X(\bar{X})}{L} \\
-\sin \Phi(\bar{X}) & -\frac{\cos \Phi(\bar{X})}{L} & -\frac{\cos \Phi(\bar{X})}{L}
\end{array}\right]}_{\mathbf{B}(\bar{X})} \underbrace{\left(\begin{array}{c}
N_{J} \\
M_{J} \\
M_{J}
\end{array}\right)}_{\mathbf{Q}}+\underbrace{\left(\begin{array}{c}
N_{q}(\bar{X}) \\
M_{q}(\bar{X}) \\
T_{q}(\bar{X})
\end{array}\right)}_{\mathbf{\Sigma}_{q}(\bar{X})}
$$

Similarly, all the integrals in Eqs. (6) to (10) are evaluated with respect to $\bar{X} \in[0 ; \bar{L}]$. The transformation of the arc-length increment $d S$ into the corresponding coordinate increment $d \bar{X}$ accomplishes the change of variable, where the quantity $\left|\mathbf{X}^{\prime}(\bar{X})\right|=\sqrt{1+(2 a \bar{X}+b)^{2}}$ is the Jacobian of the transformation between $S$ and $\bar{X}$. For instance, the element compatibility condition in Eq. (8) and flexibility matrix in Eq. (10) become:

$$
\mathbf{V}=\int_{0}^{\bar{L}} \mathbf{B}^{T}(\bar{X}) \mathbf{E}(\bar{X})\left|\mathbf{X}^{\prime}(\bar{X})\right| d \bar{X} \quad, \quad \mathbf{F}=\frac{\partial \mathbf{V}}{\partial \mathbf{Q}}=\int_{0}^{\bar{L}} \mathbf{B}^{T}(\bar{X}) \mathbf{\Upsilon}(\bar{X}) \mathbf{B}(\bar{X})\left|\mathbf{X}^{\prime}(\bar{X})\right| d \bar{X}
$$

A particular solution for the contribution due to the distributed loads can be obtained in case these are constant along the axis arc-length, that is $q_{X}(S)=\bar{q}_{X}$ and $q_{Y}(S)=\bar{q}_{Y}$. In fact, the nodal 


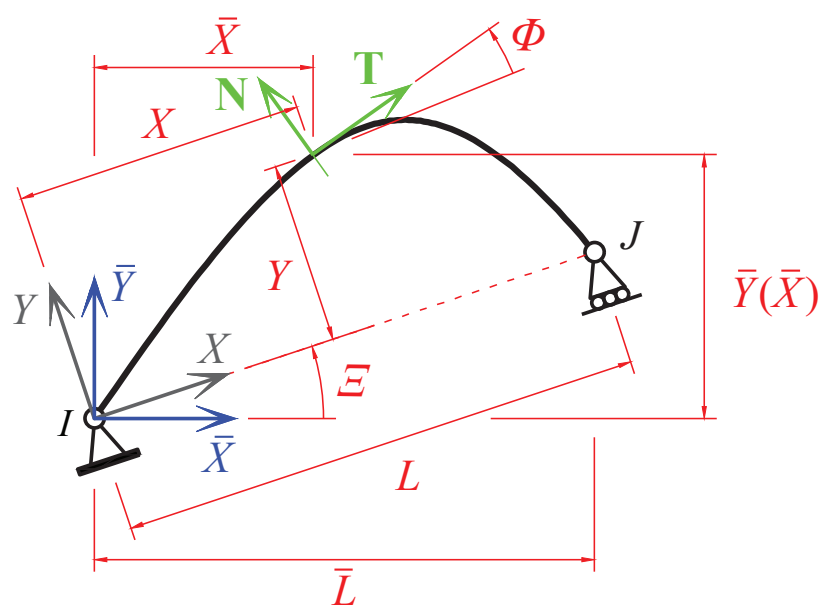

Figure 5: Geometric parametrization for the 2D curve representing the axis of a parabolic arch.

force components in Eq. (5) result:

$$
P_{X, I}^{q}=-\bar{q}_{X} L_{s} \quad, \quad P_{Y, I}^{q}=-\bar{q}_{Y}\left(L_{s}-L_{X}\right)-\bar{q}_{X} L_{Y} \quad, \quad P_{Y, J}^{q}=-\bar{q}_{Y} L_{X}+\bar{q}_{X} L_{Y}
$$

with:

$$
L_{X}=\frac{1}{L} \int_{0}^{\bar{L}} X(\bar{X})\left|\mathbf{X}^{\prime}(\bar{X})\right| d \bar{X} \quad, \quad L_{Y}=\frac{1}{L} \int_{0}^{\bar{L}} Y(\bar{X})\left|\mathbf{X}^{\prime}(\bar{X})\right| d \bar{X}
$$

and Eq. (6) simplifies to:

$$
\begin{array}{lll}
Q_{X}(\bar{X})=\bar{q}_{X} \int_{0}^{\bar{X}}\left|\mathbf{X}^{\prime}(Z)\right| d Z & , & M_{Y}(\bar{X})=\bar{q}_{X} \int_{0}^{\bar{X}}[Y(\bar{X})-Y(Z)]\left|\mathbf{X}^{\prime}(Z)\right| d Z \\
Q_{Y}(\bar{X})=\bar{q}_{Y} \int_{0}^{\bar{X}}\left|\mathbf{X}^{\prime}(Z)\right| d Z & , & M_{X}(\bar{X})=\bar{q}_{Y} \int_{0}^{\bar{X}}[X(\bar{X})-X(Z)]\left|\mathbf{X}^{\prime}(Z)\right| d Z
\end{array}
$$

The general numerical procedure consists in mapping geometric variable $\bar{X} \in[0 ; \bar{L}]$ into reference coordinate $\xi \in[-1 ;+1]$ (Fig. 6), that is:

$$
\int_{0}^{L_{s}} f(S) d S=\int_{0}^{\bar{L}} \widehat{f}(\bar{X})\left|\mathbf{X}^{\prime}(\bar{X})\right| d \bar{X}=\frac{\bar{L}}{2} \int_{-1}^{+1} \widetilde{f}(\xi)\left|\mathbf{X}^{\prime}(\xi)\right| d \xi=\frac{\bar{L}}{2} \sum_{n=1}^{n_{g}} \widetilde{f}\left(\xi_{n}\right)\left|\mathbf{X}^{\prime}\left(\xi_{n}\right)\right| w_{n}
$$

where $\widehat{f}(\bar{X})=f \circ S$ and $\widetilde{f}(\xi)=f \circ S \circ \bar{X}$. Locations of the quadrature cross-sections along $\bar{X}$ are $\bar{X}_{n}=\frac{\bar{L}}{2}\left(1+\xi_{n}\right)$; the Jacobian of the transformation between $\bar{X}$ and $\xi$ is $|J|=\frac{\bar{L}}{2}$ and, thus, $L_{n}=\frac{\bar{L}}{2}\left|\mathbf{X}^{\prime}\left(\xi_{n}\right)\right| w_{n}$ is the quadrature length associated to the $n$-th cross-section. 


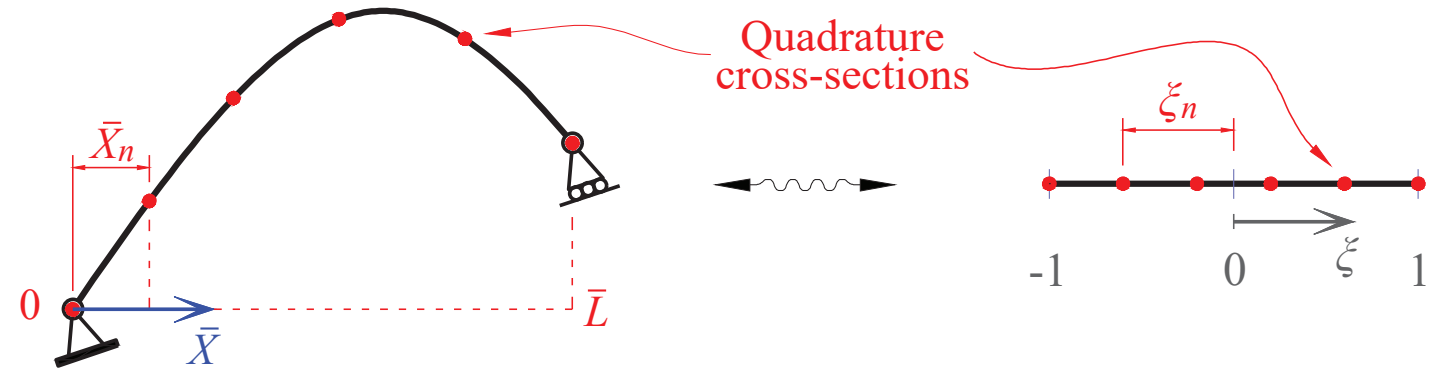

Figure 6: General numerical integration performed by mapping the coordinate abscissa $\bar{X} \in[0 ; \bar{L}]$ into the reference coordinate $\xi \in[-1 ;+1]$.

\section{Two-scale model for masonry arches}

As described in Sect. 2, the FE response in terms of nodal resisting forces $\mathbf{P}$ and element stiffness matrix $\mathbf{K}$ depends on the generalized section constitutive law (Eq. (9)), that is on the response evaluated at the $n_{g}$ quadrature cross-sections located along the element axis.

Here, a masonry arch obtained as regular, i.e periodic, sequence of bricks and mortar joints is considered. Hence, the constitutive law for masonry material and, thus, the response of the general cross-section are derived through a homogenization procedure, as described in [28]. To this end, a Unit Cell (UC) is selected as the region $\Omega$ containing a single brick $(b)$ and mortar layer $(m)$. A Cartesian reference system $(\mathrm{O}, x, y)$ is set, with $x$ aligned with curved beam tangent axis $\mathbf{T}$. Figure 7 illustrates the sketch of the UC, where $\ell_{b}$ and $\ell_{m}$ denote lengths along the UC axis of brick and mortar, respectively. As the total length of the UC, $\ell=\ell_{b}+\ell_{m}$, is assumed to be much smaller than the local curvature radius of the curved beam, a simplified parallelepiped shape is considered for the UC. Hence, a 1D straight beam model based on the Timoshenko's beam theory is used to describe UC behavior.

Generalized section strains $\mathbf{E}(S)$, i.e. quantities $E, K$ and $\Gamma$ computed at the macroscopic curved beam cross-section, are assumed as input variables to solve the Boundary Value Problem (BVP) at the microscale. At microscale level, the corresponding axial and transverse displacements, $u$ and $v$, and rotation $\theta$ of the cross-section are represented as the superposition of the kinematic map, depending on the macroscopic strains, and an unknown perturbation, as:

$$
\begin{aligned}
& u(x)=E x+u^{*}(x) \\
& v(x)=\Gamma x+\frac{1}{2} K x^{2}+v^{*}(x) \quad \text { in }(0, \ell) \\
& \theta(x)=K x+\theta^{*}(x)
\end{aligned}
$$




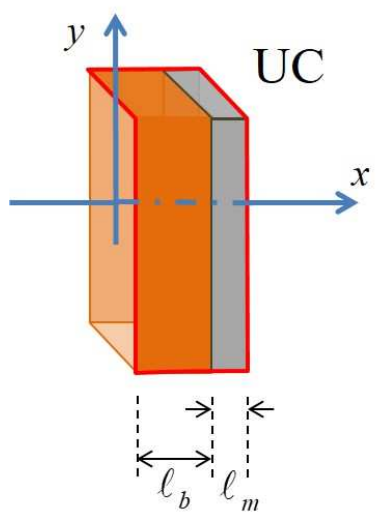

Figure 7: Unit Cell for the periodic masonry constituting masonry arch.

where $u^{*}, v^{*}$ and $\theta^{*}$ are periodic fluctuations due to $\mathrm{UC}$ heterogeneity and satisfying proper periodicity conditions on UC boundary [35]. Microscale axial, bending and shear strains are, then, obtained as:

$$
\varepsilon_{0}=E+u^{*^{\prime}} \quad, \quad \kappa=K+\theta^{*^{\prime}} \quad, \quad \gamma=\Gamma+v^{*^{\prime}}-\theta^{*}
$$

where apex $\star^{\prime}$ denotes derivative with respect to $x$ of variable $\star$.

Enforcing that the average of the periodic parts of the beam strains are zero and accounting for periodicity conditions, the following condition holds for $\theta^{*}$ [28]:

$$
\int_{\ell} \theta^{*} d x=0
$$

The generalized stresses work-conjugate to $\varepsilon_{0}, \kappa$ and $\gamma$ are denoted with $n_{b}, m_{b}$ and $t_{b}$ for brick and $n_{m}, m_{m}$ and $t_{m}$ for the mortar. A linear elastic constitutive relationship is considered for the brick, with $E_{b}$ and $G_{b}$ being the brick Young's and shear modulus, respectively. Thus, generalized stress contributions of the brick result as:

$$
n_{b}=E_{b} A \varepsilon_{0} \quad, \quad m_{b}=E_{b} I \kappa \quad, \quad t_{b}=G_{b} A_{s} \gamma
$$

where $A, I$ and $A_{s}$ are area, inertia and shear area of the beam cross-section, respectively. A nonlinear constitutive law is assumed for mortar joints for which the generalized stress-strain relationships 
result as [28]:

$$
\begin{aligned}
n_{m} & =E_{m} A \varepsilon_{0}-\int_{A} E_{m} \pi_{\varepsilon} d A \\
m_{m} & =E_{m} I \kappa+\int_{A} E_{m} y \pi_{\varepsilon} d A \\
t_{m} & =G_{m} A_{s} \gamma-\int_{A_{s}} G_{m} \pi_{\gamma} d A
\end{aligned}
$$

with $E_{m}$ and $G_{m}$ being the mortar Young's and shear moduli and $\pi_{\varepsilon}$ and $\pi_{\gamma}$ the normal and shear inelastic strains at the general point of the mortar cross-section. These are expressed as:

$$
\pi_{\varepsilon}=D H(\varepsilon) \varepsilon \quad, \quad \pi_{\gamma}=D \gamma_{p}
$$

where $D \in[0 ; 1]$ is a scalar damage variable and $\varepsilon=\varepsilon_{0}-y \kappa$ and $\gamma_{p}$ are the normal strain and shear slip at the material point. Finally, $H(\varepsilon) \varepsilon$ accounts for unilateral effect, being $H(\varepsilon)=0$ if $\varepsilon \leq 0$ and $H(\varepsilon)=1$ if $\varepsilon>0$. Damage variable $D$, according to the constitutive model proposed in [36], depends on coupling of failure mode I and II and its evolution is ruled by the following law:

$$
D=\max _{\text {history }}\left\{0, \min \left\{\frac{Y_{e q}-1}{(1-\eta) Y_{e q}}, 1\right\}\right\}
$$

with

$$
Y_{e q}=\sqrt{\left(\frac{\langle\varepsilon\rangle_{+}}{\varepsilon_{0}}\right)^{2}+\left(\frac{\gamma}{\gamma_{0}}\right)^{2}} \quad \eta=\frac{1}{\left(\langle\varepsilon\rangle_{+}\right)^{2}+\gamma^{2}}\left[\left(\langle\varepsilon\rangle_{+}\right)^{2} \frac{\varepsilon_{0} \sigma_{0}}{2 G_{c I}}+\gamma^{2} \frac{\gamma_{0} \tau_{0}}{2 G_{c I I}}\right]
$$

where $\sigma_{0}$ and $\tau_{0}$ are peak values for normal and shear stresses, respectively, and $G_{c I}$ and $G_{c I I}$ fracture energies corresponding to modes I and II. The shear slip flow $\gamma_{p}$ is ruled by the Coulomb friction evolution law:

$$
\dot{\gamma}_{p}=\dot{\lambda} \operatorname{sgn}(\tau) \quad \dot{\lambda} \geq 0 \quad \varphi \leq 0, \quad \dot{\lambda} \varphi=0,
$$

where $\lambda$ is the inelastic multiplier and $\varphi=\mu 2\left(1+\nu_{m}\right)(\varepsilon-H(\varepsilon) \varepsilon)+\left|\gamma-\gamma_{p}\right|, \mu$ being the friction parameter and $\nu_{m}$ mortar Poisson ratio.

Hence, a kinematic driven homogenization problem is formulated. Once quantities $E, K$ and $\Gamma$ are assigned to the UC, the homogenization process consists in evaluating strains $\varepsilon_{0}, \kappa$ and $\gamma$ at the microscale and the corresponding stress resultants $n_{b}, m_{b}$ and $t_{b}$ for the brick and $n_{m}, m_{m}$ and $t_{m}$ for the mortar and, thus, the overall resultants $N, M$ and $T$. To solve the micromechanical 
problem for the UC and determine strain and stress microscopic quantities, the Euler differential equilibrium equations in the brick and mortar are enforced, prescribing stress continuity conditions at the brick/mortar interface, periodicity and continuity conditions of the microscale displacements and the null average condition on the pertubation rotation. Due to the nonlinear evolution of the inelastic quantities in Eq. (31), the problem is iteratively solved, where at each iteration the analytical solution is determined. The adopted solution procedure is described in detail in [28]. Finally, the overall resultants $N, M$ and $T$ are computed as the average resultant components, i.e.:

$$
\begin{aligned}
N & =\frac{1}{\ell}\left(\int_{\ell_{b}} n_{b} d x+\int_{\ell_{m}} n_{m} d x\right) \\
M & =\frac{1}{\ell}\left(\int_{\ell_{b}} m_{b} d x+\int_{\ell_{m}} m_{m} d x\right) \\
T & =\frac{1}{\ell}\left(\int_{\ell_{b}} t_{b} d x+\int_{\ell_{m}} t_{m} d x\right)
\end{aligned}
$$

These correspond to the macroscopic curved beam generalized stresses $\Sigma$ in Eq. (9), work-conjugate

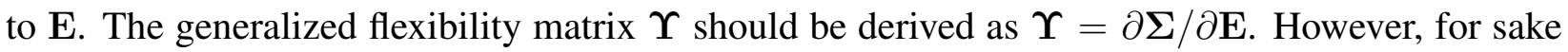
of simplicity, the initial elastic flexibility matrix $\boldsymbol{\Upsilon}_{\text {init }}$ is used here [28].

\section{Element state determination and damage regularization}

\subsection{FE solution algorithm}

The curved beam FEs formulated in Sect. 2 are implemented in a general FE program. A stepby-step time discretization is adopted, together with an iterative Newton-Raphson (N-R) algorithm for the solution of the governing nonlinear equations at each time step. As usual in FE codes, the global solution scheme requires the element state determination, that is computation of the consistent element stiffness matrix $\mathbf{K}=\partial \mathbf{P} / \partial \mathbf{U}$ and force vector $\mathbf{P}$ at each $\mathrm{N}-\mathbf{R}$ iteration.

Regardless of the coordinate parametrization for the analytical representation of the axis geometry, element state determination procedure for the implemented model coincides with that for a standard straight FB beam [11,37]. Table 1 summarizes the main steps, with the subscripts ' $k$ ' and ' $k+1$ ' denoting the previous and current N-R iteration, respectively, and $\Delta \star_{k+1}$ the current increment of the variable $\star$ with respect to the previous iteration. The described algorithm corresponds to the non-iterative procedure proposed in [11]. This permits to eliminate the nested iterative procedure usually required for element state determination of FB elements [10] and results in a remarkable computational saving. As the initial elastic flexibility matrix $\Upsilon_{\text {init }}$ is evaluated for 
Table 1: Element State Determination for Timoshenko curved beam.

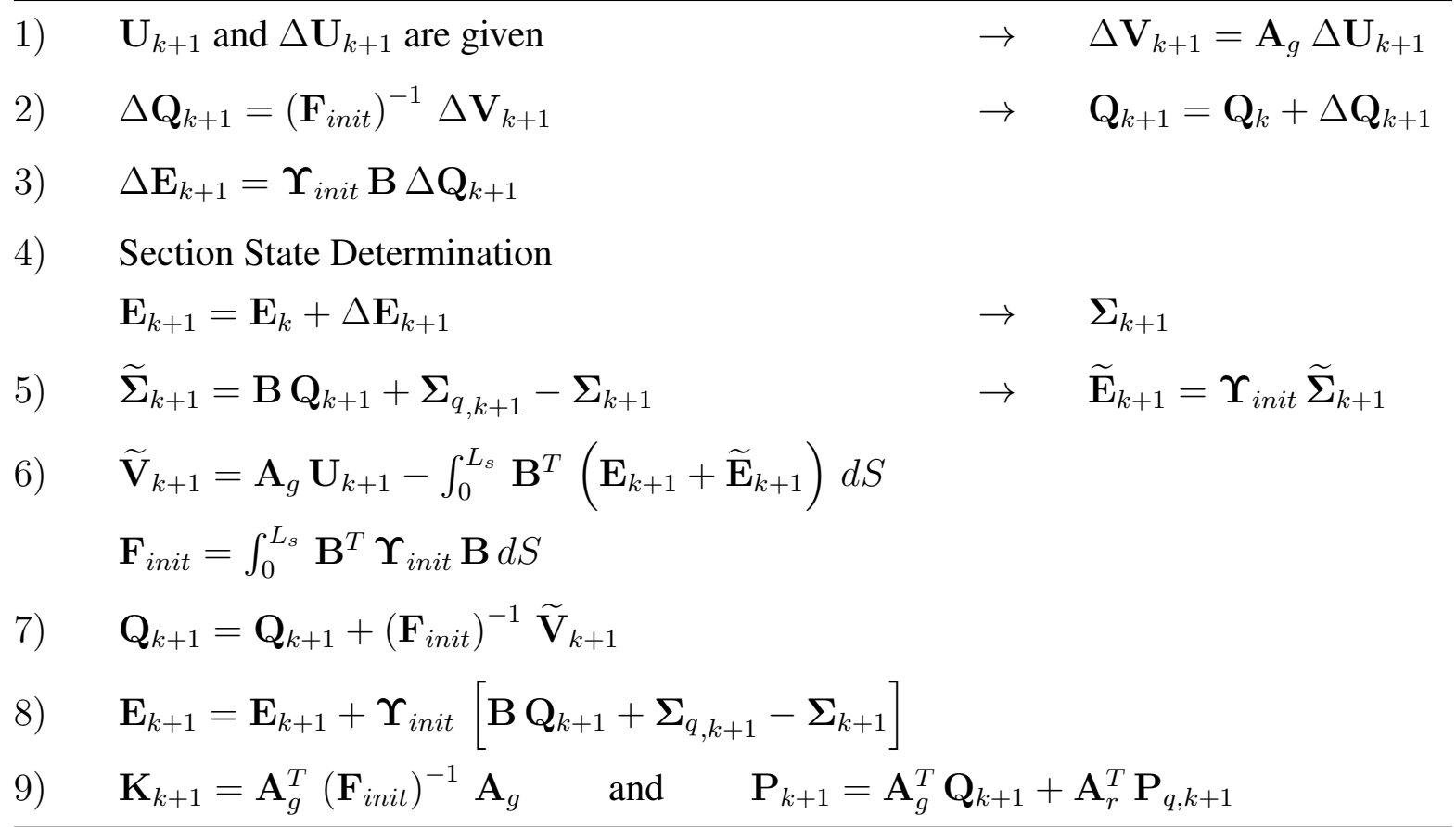

the cross-section response, the initial elastic matrices $\mathbf{F}_{\text {init }}$ and $\mathbf{K}_{\text {init }}$ are used during the whole step-by-step analysis, instead of Eq. (10) and Eq. (11).

Note that Steps 3) to 5) and Step 8) are only performed at the quadrature cross-sections used for the numerical evaluation of the integrals at Step 6). The section state determination at Step 4) consists in evaluation of the constitutive response at the quadrature points, as described in Sect. 3. Here, to solve Eq. (31) a layer discretization of the cross-section is adopted for mortar joints [28].

\subsection{Hinge detection and damage regularization approach}

When using softening materials, strain/damage localization likely occurs during the softening branch of structural response. For DB beam-column FEs, localization length coincides with the element size. For the proposed FB curved beams, localization zone corresponds to the weighting length associated to the generic quadrature cross-section [14], where damage attains the highest values. The zones where damage localizes correspond to nonlinear hinges [38] and have a primary role in element collapse mechanism.

To accurately determine hinge locations, a high number of quadrature cross-sections over each FE is here adopted. More quadrature points are used over the arch length, more locations are potentially available for the model to automatically detect hinge formation. As shown in the numerical applications (Sect. 5.3), the adoption of 20-30 quadrature points in the whole arch suffices for a re- 
alistic representation of the collapse mechanism of the structure and does not result in a particular gain of the computational effort.

However, a regularization technique is also required to overcome dependence of the numerical response on the selected number of the quadrature points [14]. This work adopts a fracture energy regularization technique, where, given the fracture energy values $G_{c I}$ and $G_{c I I}$ per unit volume for flexural and shear damaging effects, respectively (Sect. 3), the values associated to the $n$-th quadrature cross-section are evaluated as:

$$
\tilde{G}_{c I}=G_{c I} \frac{L_{c}}{L_{n}} \quad, \quad \tilde{G}_{c I I}=G_{c I I} \frac{L_{c}}{L_{n}}
$$

being $L_{c}$ a material characteristic length.

\section{Numerical applications}

To validate th 2 oposed beam model, six numerical tests are performed considering both circular and parabolic arches. The first two tests reproduce the linear elastic response of benchmarking specimens and investigate convergence properties of the FE formulation. The other tests adopt the multiscale model in Sect. 3 to simulate response of real masonry structures under damaging effects and investigate the model capability to reproduce nonlinear material response.

\subsection{Clamped-clamped linear elastic circular arch under distributed load}

The first numerical application has been originally carried out by Molari and Ubertini [27] and consists in the linear elastic analysis of the circular arch in Fig. 8. Mechanical parameters of the specimen are: Young's modulus $E=1$, shear modulus $G=0.5$, axis radius $R=1$ and square cross-section width $h=0.1$. Both the abutments are clamped and a vertical load $q=1$ is applied, uniformly distributed along horizontal direction.

To investigate the accuracy of the proposed approach, two different discretizations are considered, adopting one (mesh1) and two (mesh 2) symmetric curved FEs, respectively, based on the formulation in Sect. 2.3. Both discretizations use an increasing number $n_{g}$ of Gauss-Legendre quadrature cross-sections per each FE, whose locations $\Psi_{n}$ are defined according to Eq. (18). Horizontal reaction force in $A$ and vertical displacement in $B$ (Fig. 8) are computed and compared with exact values and numerical results in [27].

Fig. 9 shows the convergence of the solution (black curves for mesh 1 and red curves for mesh 2), plotting (a) the horizontal nodal reaction in $A$ and (b) the vertical displacement in $B$ vs total number 


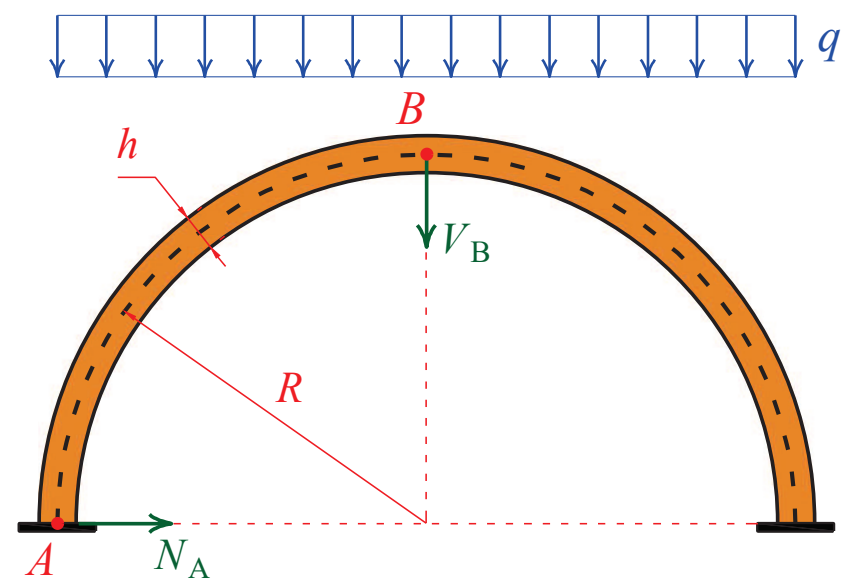

Figure 8: Clamped-clamped linear elastic circular arch under distributed load.

of quadrature cross-sections used for the whole arch. The monitoring quantities are expressed as percentage errors, that is:

$$
\text { Error }[\%]=\frac{\text { Numerical value }- \text { Exact value }}{\text { Exact value }} 100
$$

Blue lines and green stars represent the exact values and the numerical solutions reported in [27], respectively. The latter are obtained with one FB curved beam (see also Sect. 2.2), using a 5point quadrature scheme. Note that the axis geometry considered by this model is not exact and a specific algorithm is required to obtain a sufficiently accurate representation of the arch. However, the error, which is related to both approximated geometry and numerical integration, is negligible. By contrast, the model here proposed exactly describes arch geometry and the error depends only on numerical integration procedure. Five and three quadrature cross-sections per element suffice to obtain accurate results, for mesh 1 and 2 , respectively. Note that the vertical displacement $V_{B}$ in case of mesh 1 can not be obtained as nodal quantity and, thus, it is not given. Indeed, there is no node located at the key of the arch and any other evaluation of this displacement would make the comparison with the other FEs unfair.

Finally, the same figure also shows results for two additional numerical models. These discretize the arch with uniformly distributed Timoshenko straight beam FEs, based on a FB [15] (blue curves with dots) and a locking-free DB (blue curves with squares) formulation, respectively. The latter [39] considers linear shape functions for the displacement interpolation, but enforce a uniform distribution of the generalized shear strains along the axis to prevent shear-locking. For both models, the number of Gauss-Legendre quadrature cross-sections per FE is equal to $n_{g}=2$, providing exact integration of element quantities. However, the number of FEs used to discretize the arch varies. 


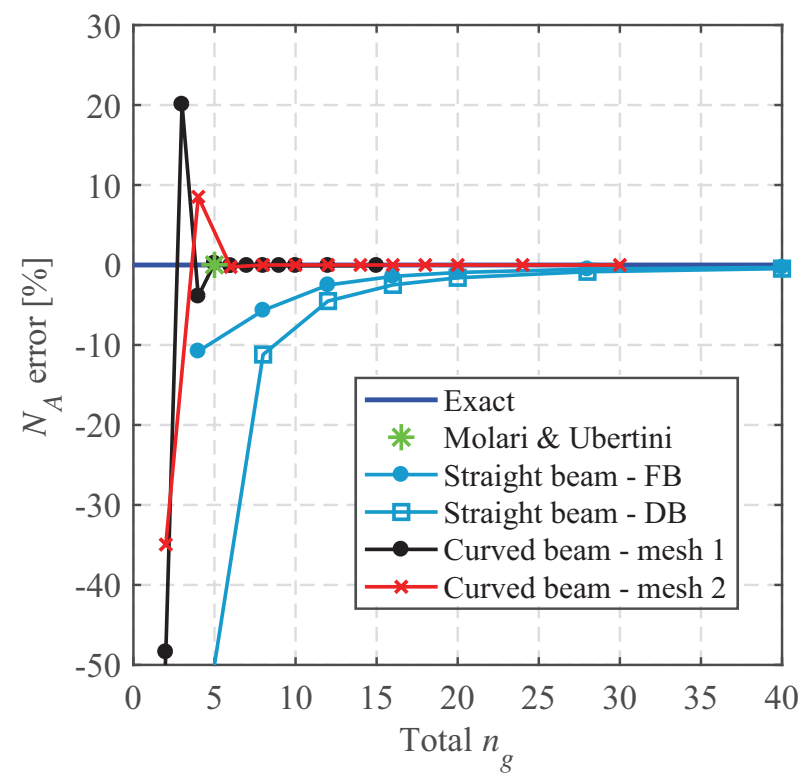

(a) Horizontal reaction in $A$.

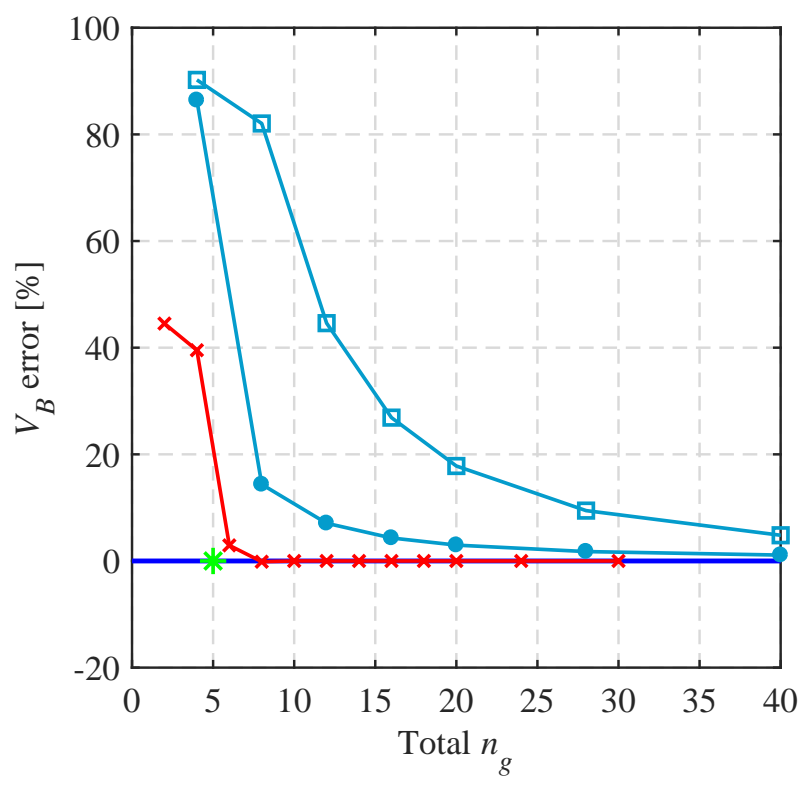

(b) Vertical displacement in $B$.

Figure 9: Convergence study for the clamped-clamped linear elastic circular arch under distributed load.

Indeed, due to geometry approximation, a lot of elements is needed to improve solution accuracy, as the rate of convergence for these models is significantly lower.

Hence, the proposed FE gives the best performances with respect to those considered for comparison. In fact, it provides a very accurate solution, similar to that obtained in [27], but exactly models arch geometry, without requiring any ad hoc procedure to describe its axis.

\subsection{Pinched ring}

To further investigate the rate of convergence of the proposed approach under linear elastic material response, the second test analyzes the pinched ring in Fig. 10 and compares results with those obtained from enhanced curved beam formulations [18, 40]. Mechanical parameters of the specimen are: Young's modulus $E=72394.9 \mathrm{MPa}$, Poisson coefficient $\nu=0.3125$, mid-line radius $R=125.8 \mathrm{~mm}$ and thickness $h=2.39 \mathrm{~mm}$. Only one quarter of the ring is modeled adopting from one to four proposed curved FEs (Sect. 2.3) with $n_{g}=3$ Gauss-Legendre quadrature cross-sections, located according to Eq. (18).

Fig. 11 shows convergence of the solution, plotting the error for displacement $V_{A}$ at the loaded point of the ring and considering a reference value of $31.60 \mathrm{~mm}[18,40]$. Two enhanced curved beam models are considered for comparison: the isoparametric quadratic element by Prathap and 


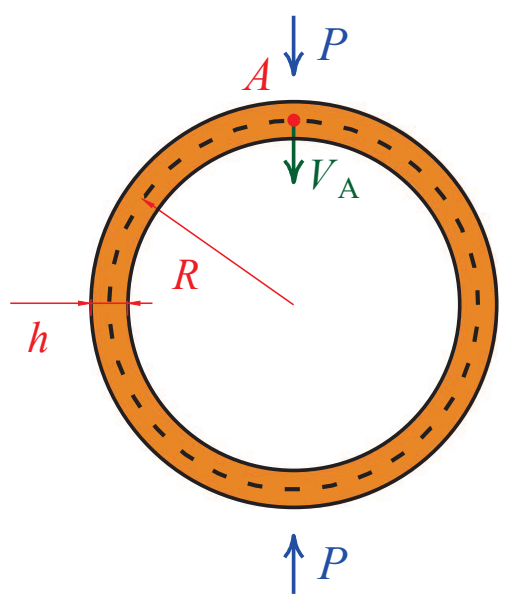

Figure 10: Pinched ring.

Babu [40] and the 3-node element with assumed strains by Choi and Lim [18]. In addition, the solution obtained with straight FB beams is reported.

As observed in the previous test, one proposed FE suffices to get the correct solution, as opposed to the comparison models. Indeed, correct definition of the axis geometry and equilibrated interpolation of the beam internal forces in Eq. (14) permit the exact definition of the element stiffness matrix, as usual in FB formulations. Numerical errors may be caused only by inaccurate quadrature schemes assumed for element quantity evaluation, that is when few quadrature points are adopted along the element axis.

\subsection{In-plane bending of a masonry circular arch}

The third test deals with the nonlinear behavior of a masonry circular arch under in-plane bending. The specimen, experimentally tested by Cancelliere et al. [41], has been numerically studied in [42] adopting the multiscale model in Sect. 3 to describe the material nonlinear response, but using seventeen straight Euler-Bernoulli FEs to discretize the structure. In the following, the curved beam in Sect. 2.3 is used to investigate performances of the formulation in nonlinear material range and study influence of the quadrature scheme adopted for the numerical integration.

Fig. 12 shows geometry of the arch, with $\ell_{b}=55 \mathrm{~mm}$ and the mortar length varying from $0.8 \mathrm{~mm}$ to $15.5 \mathrm{~mm}$; the average value $\ell_{m}=10 \mathrm{~mm}$ is assumed in the analyses. The abutments are fully restrained and a vertical force acts on the external surface at $140 \mathrm{~mm}$ from the arch vertex.

Two FEs are used to discretize the arch and the inter-elements node coincides with the point of application of the concentrated force. The quadrature scheme is based on Gauss-Lobatto integration rule, to place controlling cross-sections at element ends where some of the hinges are likely to form. 


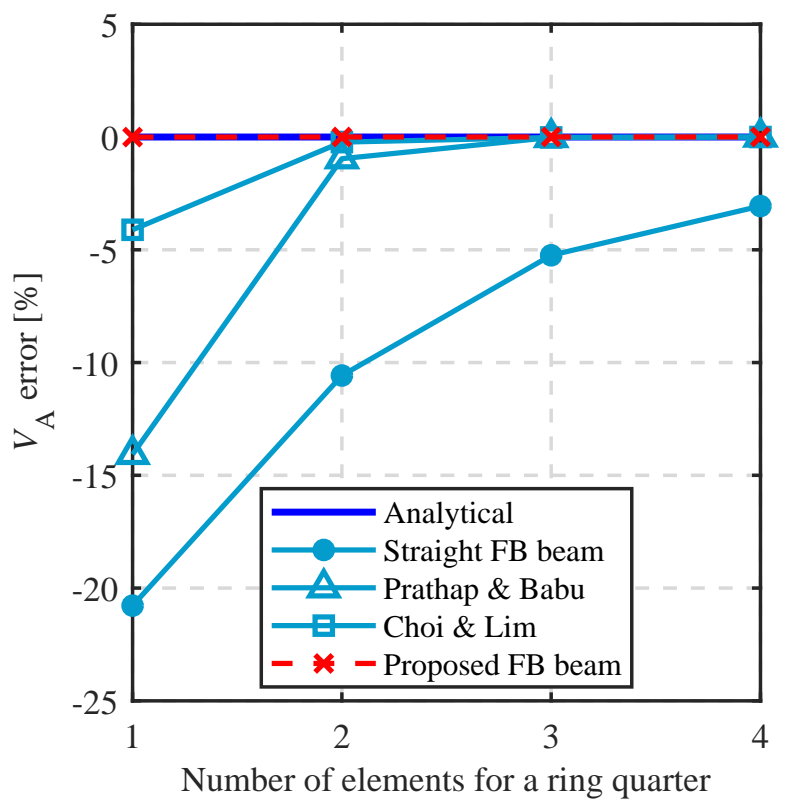

Figure 11: Convergence study for the pinched ring: comparison of different beam FE formulations.

Table 2: Material parameters for bricks and mortar of the circular arch.

\begin{tabular}{|ccccccccc|}
\hline $\begin{array}{c}E_{b} \\
{[\mathrm{MPa}]}\end{array}$ & $\begin{array}{c}G_{b} \\
{[\mathrm{MPa}]}\end{array}$ & $\begin{array}{c}E_{m} \\
{[\mathrm{MPa}]}\end{array}$ & $\begin{array}{c}G_{m} \\
{[\mathrm{MPa}]}\end{array}$ & $\begin{array}{c}\sigma_{0} \\
{[\mathrm{MPa}]}\end{array}$ & $\begin{array}{c}\tau_{0} \\
{[\mathrm{MPa}]}\end{array}$ & $\begin{array}{c}G_{c I} \\
{[\mathrm{MPa}]}\end{array}$ & $\begin{array}{c}G_{c I I} \\
{[\mathrm{MPa}]}\end{array}$ & $\begin{array}{c}\mu \\
{[-]}\end{array}$ \\
\hline 16000 & 6666.67 & 1500 & 625 & 0.005 & 1.0 & $3.0 \times 10^{-4}$ & $4.0 \times 10^{-3}$ & 0.50 \\
\hline
\end{tabular}

Hence, the locations $\Psi_{n}$ of the cross-sections are defined according to Eq. (18). Thirty layers are used over each cross-section for damage and plasticity evaluation.

Table 2 contains the material parameters deduced from the experimental measurements in [41], while the self weight of the masonry is assumed equal to $17 \mathrm{kN} / \mathrm{m}^{3}$. The experimental measurements show that debonding phenomena at interfaces mainly causes the nonlinear hinge formation, whereas limited damage and micro-cracks occur in short portions of the mortar. Hence, the characteristic length for the model regularization approach in Eq. (37) is set equal to the mortar length $(10 \mathrm{~mm})$.

Fig. 13 plots the nonlinear response of the arch in terms of applied load $F$ vs vertical displacement $V$ of the loaded cross-section. Red lines in Fig. 13(a) refer to the numerical results obtained with the proposed Two-Scale 1D model adopting different values of $n_{g}$ and blue dots denote the experimental data. As the numerical model evaluates cross-section response only at the quadrature points, their locations coincide with those of the hinges potentially detected by the model. Hence, a value of $n_{g}$ greater than 10 is necessary to obtain an accurate solution. Indeed, the best match with 

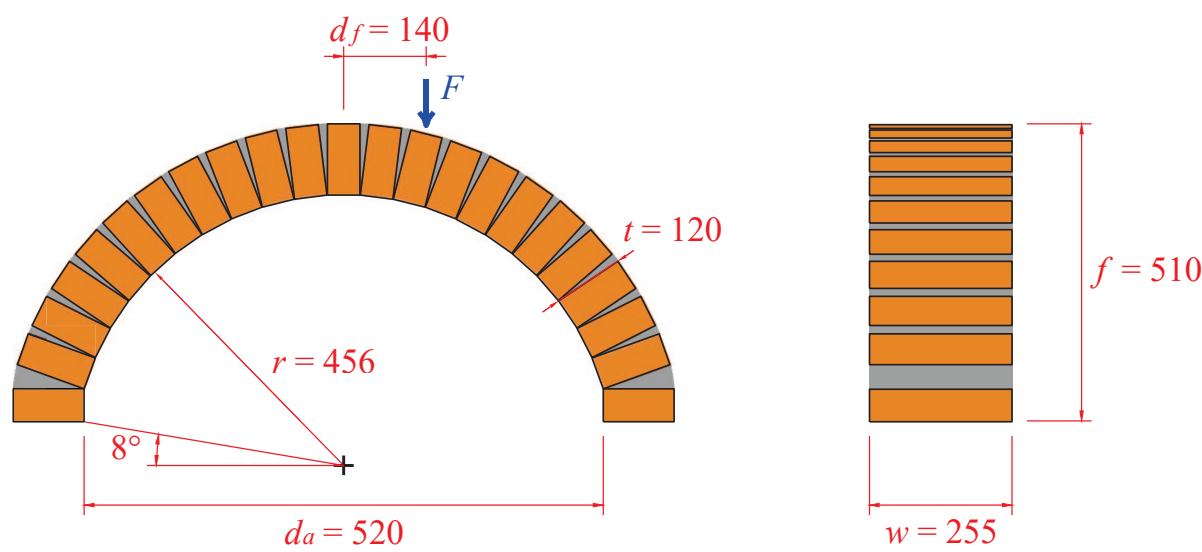

Figure 12: Geometry of the masonry circular arch (dimensions in $\mathrm{mm}$ ).

the experimental data results by placing quadrature cross-sections exactly where the hinges form in real test. Thus, increasing $n_{g}$ increases number of points where hinges can potentially form. Fig. 13(b) compares the solution for $n_{g}=13$ (red line) with the failure load of $650 \mathrm{~N}$ deduced from the limit analysis and the results of the micromechanical 2D approach proposed by Addessi et al. [8] (green line). The curves show a good match between the different approaches, although the proposed model exhibits a slightly lower strength.

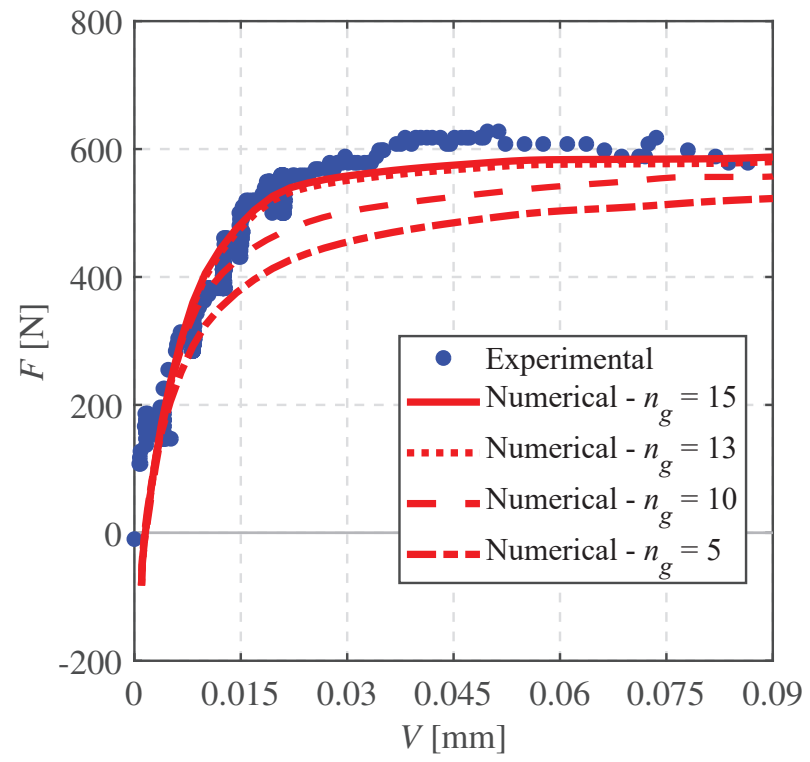

(a) Convergence study for variable $n_{g}$.

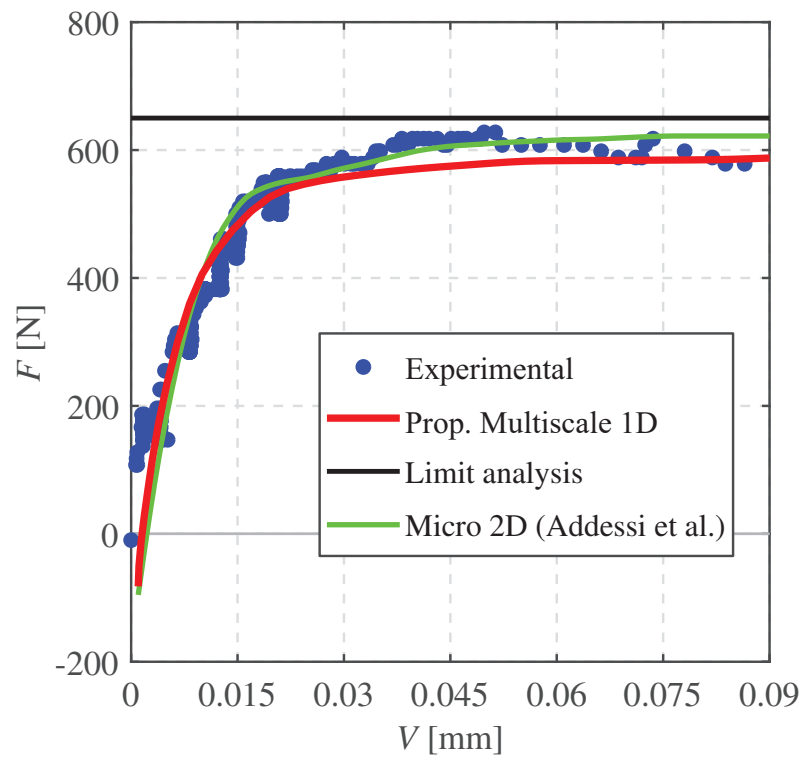

(b) Comparison with micro 2D model and limit analysis.

Figure 13: Nonlinear response of the masonry circular arch: applied force $F$ versus vertical displacement $V$ of the loaded section.

Fig. 14(a) shows the locations of the hinges. Four hinges form during the experimental test: 


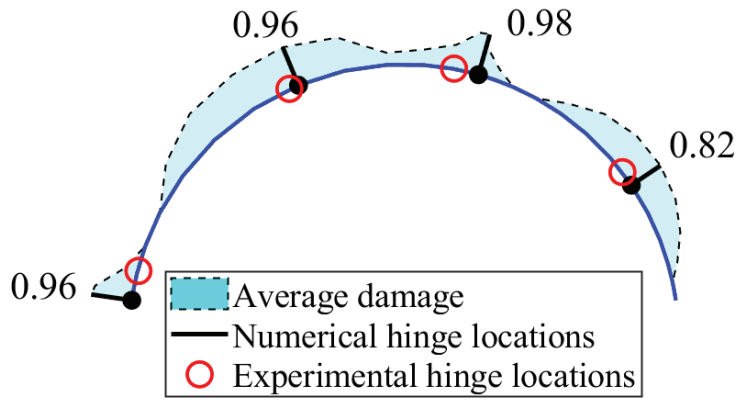

(a) Hinge locations and distribution of $D_{m}$.

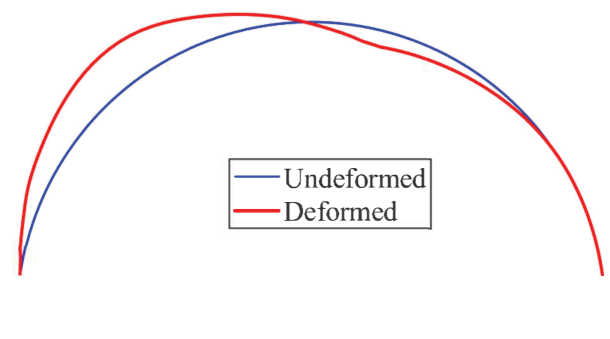

(b) Deformed configuration (scale $=300)$.

Figure 14: Masonry circular arch: (a) hinge locations and (b) deformed configuration for $V=$ $0.8 \mathrm{~mm}$.

two on intrados and two on extrados of the arch, due to decohesion between mortar and brick. Red circles indicate the locations experimentally observed; black dots, almost coinciding with red circles, denote the locations obtained by the numerical model with $n_{g}=13$. These correspond to the four cross-sections where damage variable $D$ attains highest values. The shaded area represents the average of the damage variable $D$ over each beam cross-section, defined as $D_{m}=\frac{1}{A} \int_{A} D d A$. Finally, Fig. 14(b) shows the deformed configuration of the arch for the same vertical displacement $V=0.08 \mathrm{~mm}$ of the loaded cross-section obtained from the numerical model. This accurately reproduces experimental outcomes in [41] showing a movement of the top of the arch on the lefthand side of the force, due to load eccentricity and hinge formation.

\subsection{Softening response of circular and catenary-shape arches}

To further investigate the efficiency of the regularization technique adopted to overcome damage localization problems, two additional tests are conducted. These reproduce the nonlinear response of a circular and a catenary-shape arch under in-plane bending characterized by pronounced softening behavior. Both arches have been numerically studied in [43], basing on the experimental tests reported in $[44,45]$ on identical reinforced specimens.

Fig. 15 shows arch geometries and indicates the position of the concentrated vertical force applied to the specimens, which monotonically increases until specimen failure. The other geometric parameters are arch width, $w$, and brick and mortar lengths, $\ell_{b}$ and $\ell_{m}$. These are set equal to $w=450 \mathrm{~mm}, \ell_{b}=25 \mathrm{~mm}$ and $\ell_{m}=10 \mathrm{~mm}$ for the circular arch and $w=1000 \mathrm{~mm}, \ell_{b}=125 \mathrm{~mm}$ and $\ell_{m}=10 \mathrm{~mm}$ for the catenary-shape arch.

Two and three FEs based on the formulations in Sect. 2.3 and Sect. 2.4 are used to discretize 


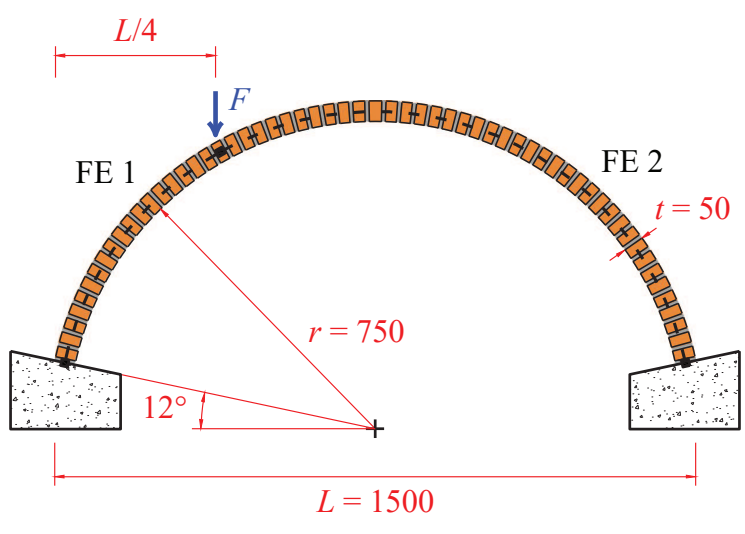

(a) Circular arch.

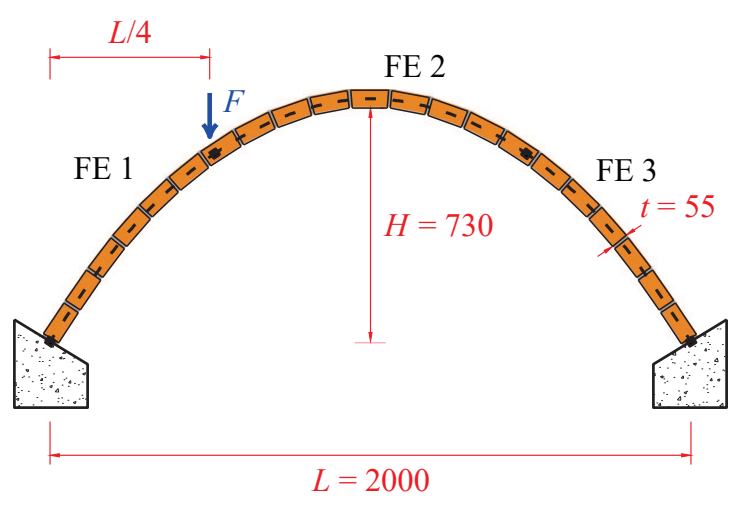

(b) Catenary-shape arch.

Figure 15: Geometry and discretization of the circular and catenary-shape arch with pronounced softening behavior (dimensions in $\mathrm{mm}$ ).

Table 3: Material parameters for bricks and mortar of the (a) circular and (b) catenary-shape arch with pronounced softening behavior.

\begin{tabular}{|c|ccccccccc|}
\hline Arch & $\begin{array}{c}E_{b} \\
{[\mathrm{MPa}]}\end{array}$ & $\begin{array}{c}G_{b} \\
{[\mathrm{MPa}]}\end{array}$ & $\begin{array}{c}E_{m} \\
{[\mathrm{MPa}]}\end{array}$ & $\begin{array}{c}G_{m} \\
{[\mathrm{MPa}]}\end{array}$ & $\begin{array}{c}\sigma_{0} \\
{[\mathrm{MPa}]}\end{array}$ & $\begin{array}{c}\tau_{0} \\
{[\mathrm{MPa}]}\end{array}$ & $\begin{array}{c}G_{c I} \\
{[\mathrm{MPa}]}\end{array}$ & $\begin{array}{c}G_{c I I} \\
{[\mathrm{MPa}]}\end{array}$ & $\begin{array}{c}\mu \\
{[-]}\end{array}$ \\
\hline (a) & 5000 & 2083.3 & 1200 & 500 & 0.25 & 0.25 & 0.0075 & 0.00125 & 0.5774 \\
(b) & 8600 & 3583.3 & 1500 & 625 & 0.40 & 0.40 & 0.0030 & 0.00300 & 0.5774 \\
\hline
\end{tabular}

the circular and catenary-shape arch, respectively, as indicated in Fig. 15 by the dashed black lines. Indeed, one node is necessary to apply the concentrated force in both tests, while an additional element is required for the catenary-shape arch to correctly approximate the axis geometry with the proposed parabolic FEs. Each FE considers a Gauss-Lobatto quadrature rule, according to Eqs. (18) and (26).

Table 3 contains the material parameters of the multiscale model in Sect. 3 adopted for the two tests. These are deduced from the experimental data in [44, 45] and from numerical test on the mortar joints reported in [43]. The characteristic length for the model regularization approach in Eq. (37) is set equal to the mortar length.

Fig. 16 plots the global response curves of the arches in terms of applied load $F$ vs vertical displacement $V$ of the loaded cross-section. Red lines refer to the numerical results obtained with the proposed Two-Scale 1D model for an increasing number $n_{g}$ of quadrature cross-sections along each FE. For the circular arch, blue curves denote the experimental data referred to two similar specimens, labelled as US1 and US2. For the catenary-shape arch, experimental data are not available. Hence, blue curves denote the numerical results in [43] obtained with two different numerical 
models and labelled as Set II and Set III. For both arches, the proposed model accurately reproduces the peak strength of the specimens. A small error emerges only for the circular arch, when $n_{g}=5$. As observed experimentally and in the numerical simulations in [43], sudden drops of the strength occur during response the post-peak branch. These correspond to abrupt formation of the nonlinear hinges and, thus, to loading steps where damage localizes at specific cross-sections of the arch. Increasing the number of quadrature points does not prevent sudden drops to occur, even though numerical accuracy is increased. Similar to the circular arch in Sect. 5.3, values of $n_{g}$ greater than 10 are necessary to obtain a satisfactory solution and to observe a correct evolution of the nonlinear hinges. Moreover, for $n_{g}>10$ the post-peak response does not depend on the number of quadrature sections located along the axis, indicating that the proposed regularization technique efficiently avoids mesh sensitivity issues.

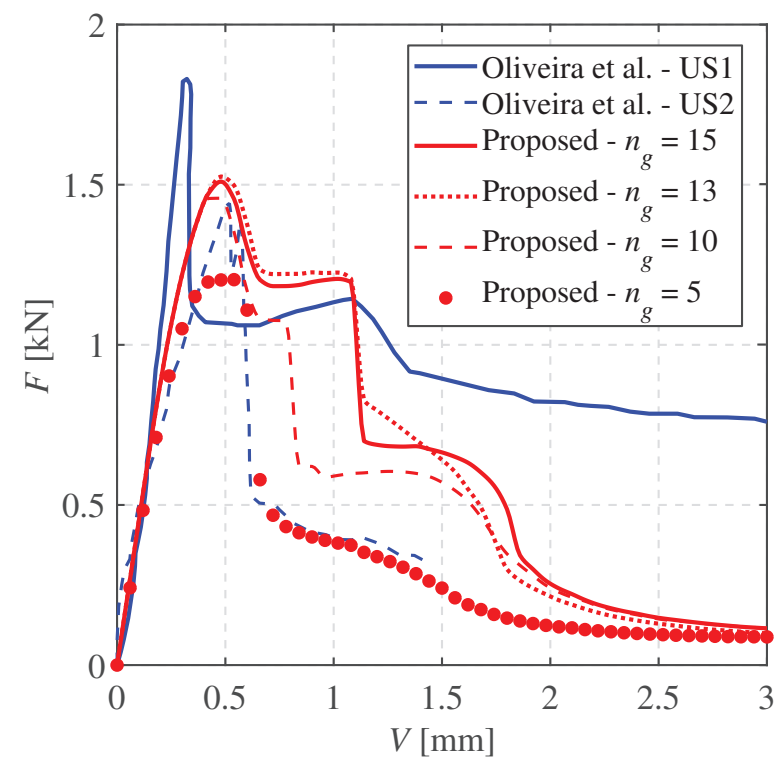

(a) Circular arch.

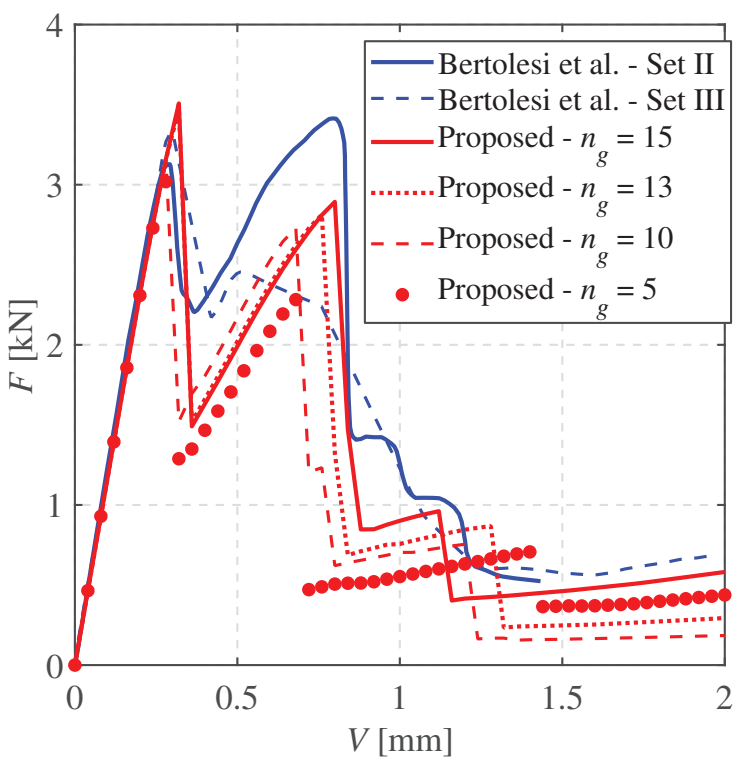

(b) Catenary-shape arch.

Figure 16: Nonlinear response of the circular and catenary-shape arch with pro 2 nced softening behavior: applied force $F$ versus vertical displacement $V$ of the loaded section.

The hinge locations can be observed from the deformed configurations plotted in Fig. 17, where deformed positions of the bricks reported in [43] (blue rectangles) are compared with deformed shapes of the arch axis obtained from the proposed beam model. One hinge forms at the load position, another on its right and two less evident hinges almost at the abutments. A very good match between proposed and reference models can be observed. 


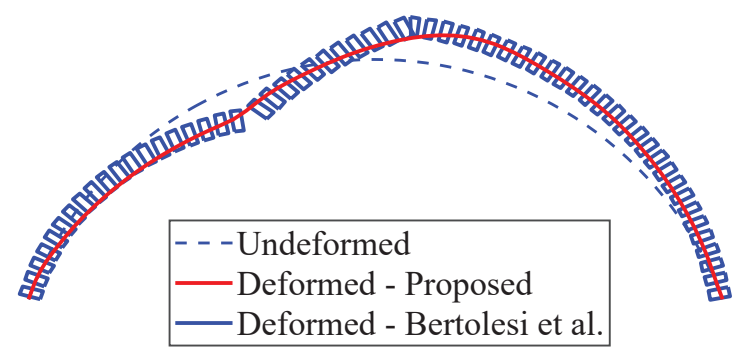

(a) Circular arch.

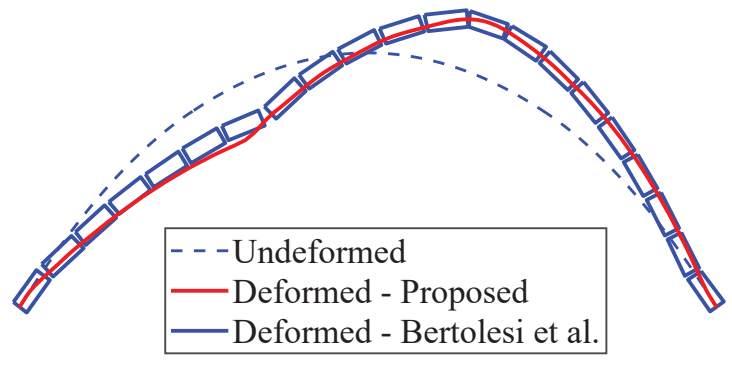

(b) Catenary-shape arch.

Figure 17: Deformed configuration for (a) circular and (b) catenary-shape arch.

\subsection{Bending of a masonry parabolic arch due to multiple in-plane forces}

Last test reproduces nonlinear response of the rectangular parabolic arch experimentally studied during the campaign by Vermeltfoort [46]. Fig. 18 shows arch geometry, having clear span $L=3 \mathrm{~m}$ and sagitta $H=0.5 \mathrm{~m}$. The axis length is equal to fifty-one brick/mortar layers, with brick length $\ell_{b}=52 \mathrm{~mm}$ and mortar length almost constant and set equal to $\ell_{m}=12 \mathrm{~mm}$ for the analyses. Thickness $t=100 \mathrm{~mm}$ and width $w=1.25 \mathrm{~m}$ are the other geometric parameters of the arch. The abutments are simply supported and steel truss elements take horizontal thrust. Four vertical
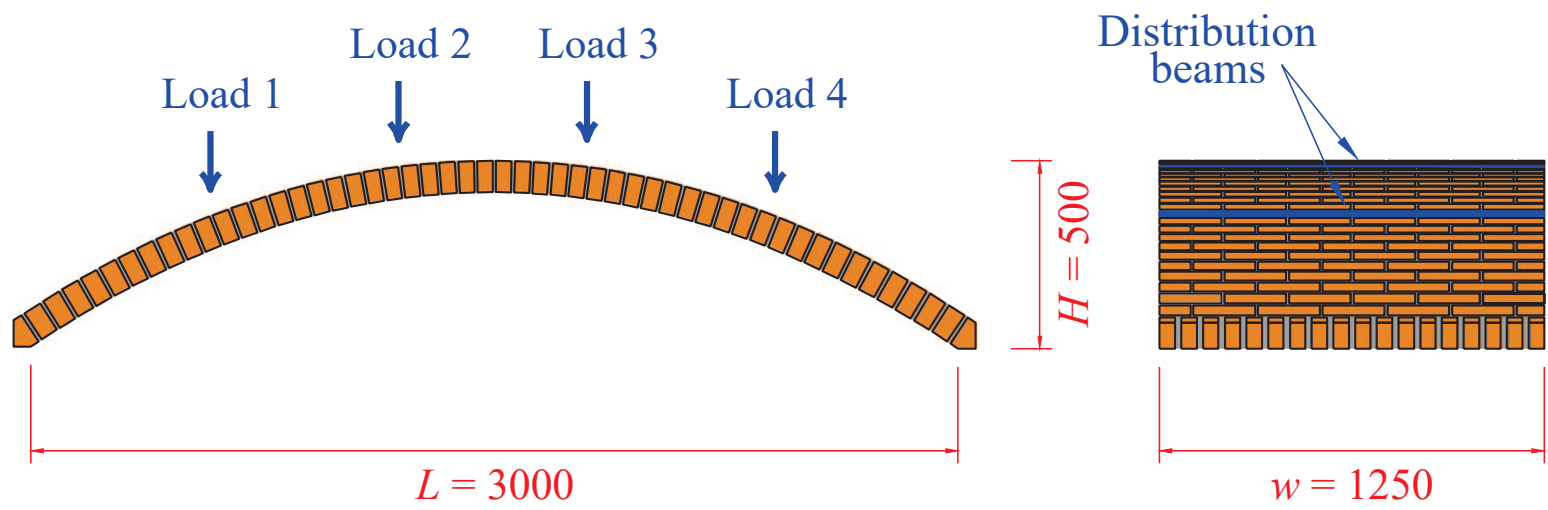

Figure 18: Geometry of the masonry parabolic arch (dimensions in $\mathrm{mm}$ ).

forces are applied on the external surface via load distribution beams parallel to the arch width and uniformly spaced in the horizontal direction. After two or three loading/unloading cycles with all the forces reaching the same maximum value of $5 \mathrm{kN}$, the final cycle considers a loading history consisting of two phases: (A) all the loads reach $5 \mathrm{kN}$; (B) only load 2 is increased until the specimen failure. The peak value for load 2 is $40.7 \mathrm{kN}$. Due to the arch deformations, during phase 
Table 4: Material parameters for bricks and mortar of the parabolic arch.

\begin{tabular}{|ccccccccc|}
\hline $\begin{array}{c}E_{b} \\
{[\mathrm{MPa}]}\end{array}$ & $\begin{array}{c}G_{b} \\
{[\mathrm{MPa}]}\end{array}$ & $\begin{array}{c}E_{m} \\
{[\mathrm{MPa}]}\end{array}$ & $\begin{array}{c}G_{m} \\
{[\mathrm{MPa}]}\end{array}$ & $\begin{array}{c}\sigma_{0} \\
{[\mathrm{MPa}]}\end{array}$ & $\begin{array}{c}\tau_{0} \\
{[\mathrm{MPa}]}\end{array}$ & $\begin{array}{c}G_{c I} \\
{[\mathrm{MPa}]}\end{array}$ & $\begin{array}{c}G_{c I I} \\
{[\mathrm{MPa}]}\end{array}$ & $\begin{array}{c}\mu \\
{[-]}\end{array}$ \\
\hline 5000 & 2500 & 1200 & 800 & 0.32 & 1.0 & 1.1 & 0.55 & 0.37 \\
\hline
\end{tabular}

Table 5: Loading history for the masonry parabolic arch.

\begin{tabular}{|c|cccc|}
\hline Phase & Load 1 & Load 2 & Load 3 & Load 4 \\
\hline $\mathrm{A}$ & $0 \mathrm{kN} \rightarrow 5 \mathrm{kN}$ & $0 \mathrm{kN} \rightarrow 5 \mathrm{kN}$ & $0 \mathrm{kN} \rightarrow 5 \mathrm{kN}$ & $0 \mathrm{kN} \rightarrow 5 \mathrm{kN}$ \\
& Force control & Force control & Force control & Force control \\
B & $5 \mathrm{kN} \rightarrow 5.9 \mathrm{kN}$ & $5 \mathrm{kN} \rightarrow$ Collapse & $5 \mathrm{kN} \rightarrow 9.1 \mathrm{kN}$ & $5 \mathrm{kN} \rightarrow 9.1 \mathrm{kN}$ \\
& Force control & Displacement control & Force control & Force control \\
\hline
\end{tabular}

B, loads 1, 3 and 4 slightly increase as well: at the peak of load 2, load 1 is equal to $5.9 \mathrm{kN}$ and loads 3 and 4 are both equal to $9.1 \mathrm{kN}$.

Five FEs based on the formulation in Sect. 2.4 are used to discretize the arch, as four nodes are necessary to apply the concentrated forces. In each FE, five Gauss-Lobatto quadrature crosssections are located with a twenty-five layer discretization scheme for damage and plasticity evaluation. Locations $\bar{X}_{n}$ of the element cross-sections are defined according to Eq. (26).

Table 4 contains the material parameters of the multiscale model in Sect. 3 adopted for the two arches. Elastic moduli of bricks and mortar, tensile and shear strengths and friction angle are deduced from [47]; fracture energies are calibrated according to the regularization technique in Sect. 4.2. As for the masonry circular arch, the characteristic length for the model regularization approach in Eq. (37) is set equal to the mortar length.

Fig. 19 plots the numerical response obtained for the two phases of the final load cycle. The curves (blue dots for the experimental data and red lines for the numerical outcomes) plot the total load in the four points vs vertical displacements $V$ of the point of application of load 2. Up to $4 \times 5=20 \mathrm{kN}$ loads are applied according to a standard force controlled procedure; then, displacement $V$ is controlled until specimen failure, while load 1, 3 and 4 are linearly increased until their peak values, (see Table 5). As shown, numerical responses perfectly agree with the real response of the arch. Solid red line refers to the proposed Two-Scale 1D (Multiscale) model, whereas dotted red line refers to a micromechanical 1D (Micro) model, where bricks and mortar layers are modeled with linear elastic and nonlinear straight beams based on the same constitutive relationship of the Multiscale model, respectively. For comparison, the solutions obtained through two different limit analysis approaches are plotted, represented by the two horizontal blue lines. 


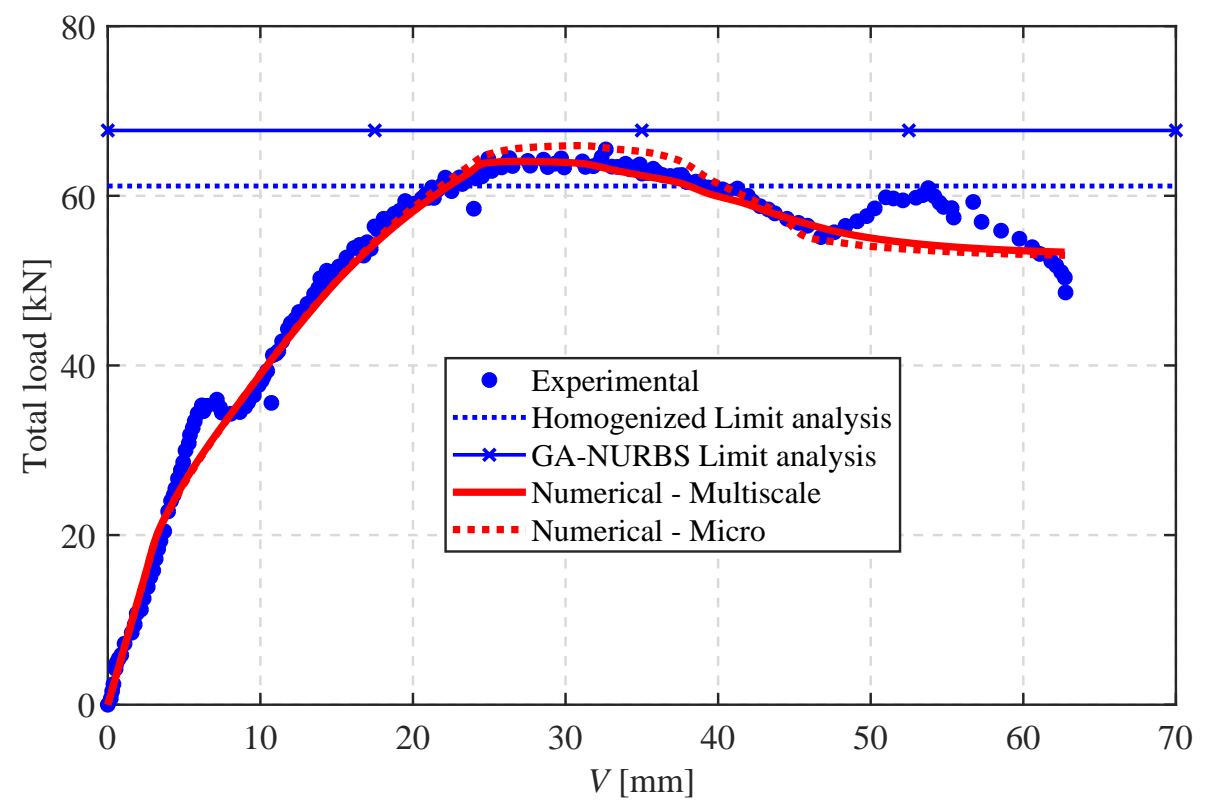

Figure 19: Global response curve for the masonry parabolic arch.

These values are reported in [48] referring to the maximum value of load 2. To compare them with the results in Fig. 19, the peak values of load 1, 3 and 4 have been added.

During phase B, two hinges form and produce arch collapse: one located at load 2 and the other on the left-hand side of load 4 . Also in this case, the proposed multiscale model correctly detects the hinge locations, as shown in Fig. 20(a). The figure compares the experimentally observed hinges (red circles) with those obtained by the numerical model (black dots) at the peak load. The shaded area represents the average damage $D_{m}$, assuming values 0.99 and 0.79 at the hinges.

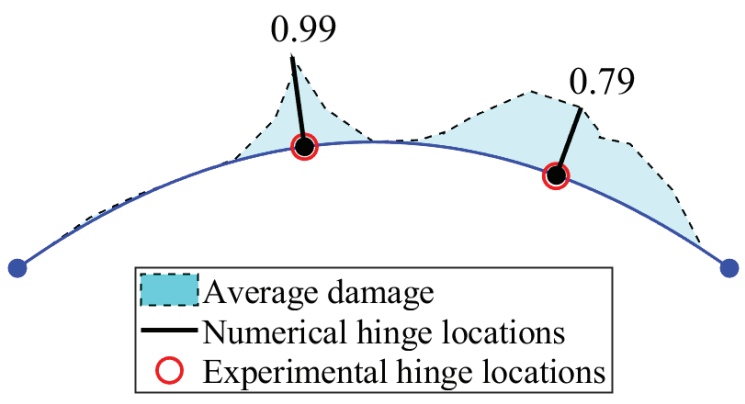

(a) Hinge locations and distribution of $D_{m}$.

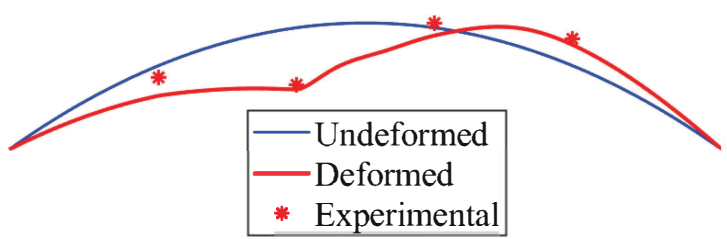

(b) Deformed configuration (scale $=10$ ).

Figure 20: Masonry parabolic arch: (a) hinge locations and (b) deformed configuration at peak load.

Finally, Fig. 20(b) shows the corresponding deformed configuration, obtained by neglecting the 
horizontal displacements of the cross-sections, not experimentally available. Red stars represent the experimental displacements of the loaded cross-sections. These are accurately reproduced by the numerical model that shows drop of the left-hand side of the arch and raising of the right-hand side.

\section{Concluding remarks}

This paper has introduced a 2D Timoshenko curved beam FE for the nonlinear analysis of periodic masonry arches. The model is based on a two-scale homogenization procedure, where the response of the curved element at the macro-scale is derived from the homogenized response of UCs at the mircoscale. These are linked to the element quadrature cross-sections and are modeled as straight beams. Bricks are considered as elastic, while a damage-friction model is used to reproduce mortar behavior. The curved axis geometry of the arch is exactly described, adopting the appropriate parametrization of the corresponding 2D curve. Specific quadrature techniques are described for each parametrization, also exploited to detect formation of the hinges due to nonlinear mechanisms.

Six numerical tests were performed to validate the proposed formulation and investigate the efficiency of the model under both linear elastic and nonlinear material responses. The two linear elastic tests prove the superior capacities of the proposed FB approach with respect to classic FEs and existing proposals, as it provides almost exact solutions in terms of all the static and kinematic variables involved in the problem, yet requiring a lower computational cost. The simulation of four experimental masonry arches subjected to in-plane bending shows that the proposed model is robust and accurate under nonlinear material responses as well. The adopted two-scale homogenization permits a satisfactory description of the nonlinear damage-plastic phenomena occurring in the mortar layers and at brick-mortar interfaces and the resulting hinge evolution is correctly reproduced by the numerical model. Comparison with experimental outcomes and additional micromechanical numerical models confirms these results, showing that the proposed FE is able to reproduce the global response of the specimens as well as the location of the hinges along the axis and the deformed configuration at all the stages of the loading process. Finally, the two-scale beam formulation presented is an efficient and fast tool, compared to more sophisticated 2D and 3D nonlinear FE models, that can be easily generalized to analyze more interesting but complex structural problems, such as strengthened masonry arches and walls [43]. 


\section{References}

[1] F. Freddi, E. Sacco, and R. Serpieri. An enriched damage-frictional cohesive-zone model incorporating stress multi-axiality. Meccanica, 2017. doi: 10.1007/s11012-017-0777-z.

[2] A. Gaetani, G. Monti, P. B. Lourenço, and G. Marcari. Design and analysis of cross vaults along history. Int J Archit Herit, 10(7):841-856, 2016. doi: 10.1080/15583058.2015.1132020.

[3] G. Milani and P. B. Lourenço. 3D non-linear behavior of masonry arch bridges. Comput Struct, 110-111:133-150, 2012. doi: 10.1016/j.compstruc.2012.07.008.

[4] A. Iannuzzo, M. Angelillo, E. De Chiara, F. De Guglielmo, F. De Serio, F. Ribera, and A. Gesualdo. Modelling the cracks produced by settlements in masonry structures. Meccanica, 2017. doi: 10.1007/s11012-017-0721-2.

[5] C. Molins and R. Pere. Capacity of masonry arches and spatial frames. J Struct Eng - ASCE, 124(6):653-663, 1998.

[6] A. Thavalingam, N. Bicanic, J. I. Robinson, and D. A. Ponniah. Computational framework for discontinuous modelling of masonry arch bridges. Comput Struct, 79(19):1821-1830, 2001. doi: 10.1016/S0045-7949(01)00102-X.

[7] P. Roca, M. Cervera, G. Gariup, and L. Pelà. Structural analysis of masonry historical constructions. classical and advanced approaches. Arch Comput Methods Eng, 17(3):299-325, 2010. doi: 10.1007/s11831-010-9046-1.

[8] D. Addessi, S. Marfia, E. Sacco, and J. Toti. Modeling approaches for masonry structures. The Open Civil Engineering Journal, 8:288-300, 2014. doi: 10.1016/j.ijsolstr.2011.12.001.

[9] T. J. Massart, R. H. J. Peerlings, and M. G. D. Geers. Structural damage analysis of masonry walls using computational homogenization. Int J Damage Mech, 16(2):199-226, 2007. doi: 10.1007/s11831-010-9046-1.

[10] E. Spacone, V. Ciampi, and F. C. Filippou. A beam element for seismic damage analysis. Earthquake Engineering Research Center, University of California, 1992.

[11] A. Neuenhofer and F. C. Filippou. Evaluation of nonlinear frame finite-element models. $J$ Struct Eng - ASCE, 123(7):958-966, 1997. 
[12] B. N. Alemdar and D. W. White. Displacement, flexibility, and mixed beam-column finite element formulations for distributed plasticity analysis. Struct Eng, 131(12):1811-1819, 2005. doi: 10.1061/(ASCE)0733-9445(2005)131:12(1811).

[13] E. Spacone, F. C. Filippou, and F. F. Taucer. Fibre beam-column model for non-linear analysis of R/C frames: Part 1. Formulation. Earthquake Eng Struct, 25:711-725, 1996.

[14] D. Addessi and V. Ciampi. A regularized force-based beam element with a damage-plastic section constitutive law. Int J Numer Meth Engng, 70:610-629, 2007. doi: 10.1002/nme.1911.

[15] A. Saritas and F. C. Filippou. Inelastic axial-flexure-shear coupling in a mixed formulation beam finite element. Int J Nonlinear Mech, 44(8):913-922, 2009. doi: 10.1016/j.ijnonlinmec.2009.06.007.

[16] P. Di Re, D. Addessi, and F. C. Filippou. Mixed 3D beam element with damage plasticity for the analysis of RC members under warping torsion. J Struct Eng, 144(6):04018064, 2018. doi: 10.1061/(ASCE)ST.1943-541X.0002039.

[17] J.N. Reddy. On locking-free shear deformable beam finite elements. Computer Methods in Applied Mechanics and Engineering, 149(1):113 - 132, 1997. ISSN 0045-7825. doi: https://doi.org/10.1016/S0045-7825(97)00075-3. Containing papers presented at the Symposium on Advances in Computational Mechanics.

[18] J.-K. Choi and J-K. Lim. General curved beam elements based on the assumed strain fields. Comput Struct, 55(3):379 - 386, 1995. ISSN 0045-7949. doi: https://doi.org/10.1016/00457949(95)98865-N.

[19] A. Benedetti and A. Tralli. A new hybrid F.E. model for arbitrarily curved beam-I. Linear analysis. Comput Struct, 33(6):1437-1449, 1989. doi: 10.1016/0045-7949(89)90484-7.

[20] A. Krishnan and Y. J. Suresh. A simple cubic linear element for static and free vibration analyses of curved beams. Comput Struct, 68(5):473-489, 1998. doi: 10.1016/S00457949(98)00091-1.

[21] P. Raveendranath, G. Singh, and B. Pradhan. A two-noded locking-free shear flexible curved beam element. Int J Numer Meth Engng, 44(2):265-280, 1999.

[22] J. S. Wu and L. K. Chiang. Free vibration analysis of arches using curved beam elements. Int J Numer Meth Engng, 58(13):1907-1936, 2003. 
[23] M. Cannarozzi and L. Molari. A mixed stress model for linear elasto dynamics of arbitrarily curved beams. Int J Numer Meth Engng, 74:116-137, 2008. doi: 10.1002/nme.2161.

[24] M. Cannarozzi and L. Molari. Stress-based formulation for non-linear analysis of planar elastic curved beams. Int J Nonlinear Mech, 55:35-47, 2013. doi: 10.1016/j.ijnonlinmec.2013.04.005.

[25] B. L. Koziey and F. A. Mirza. Consistent curved beam element. Comput Struct, 51(6):643654, 1994.

[26] Y. L. Pi and M. A. Bradford. Elasto-plastic buckling and postbuckling of arches subjected to a central load. Comput Struct, 81(18):1811-1825, 2003.

[27] L. Molari and F. Ubertini. A flexibility-based finite for linear analysis of arbitrarily curved arches. Int J Numer Meth Engng, 65(8):1333-1353, 2006. doi: 10.1002/nme.1497.

[28] D. Addessi and E. Sacco. Homogenization of heterogeneous masonry beams. Meccanica, 53: 1699-1717, 2018. doi: 10.1007/s11012-017-0758-2.

[29] R. Peerlings, M. Geers, R. De Brost, and W. Brekelmans. A critical comparison of nonlocal and gradient-enhanced softening continua. Int J Solids Struct, 38(44):7723-7746, 2001. doi: 10.1016/S0020-7683(01)00087-7.

[30] C. Comi and U. Perego. Fracture energy based bi-dissipative damage model for concrete. Int J Solid Struct, 38(36):6427-6454, 2001.

[31] M. H. Scott and O. M. Hamutçuoğlu. Numerically consistent regularization of force-based frame elements. Int J Numer Meth Engng, 76:1612-1631, 2008. doi: 10.1002/nme.2386.

[32] P. Di Re and D. Addessi. A mixed 3D corotational beam with cross-section warping for the analysis of damaging structures under large displacements. Meccanica, 53(6):1313-1332, 2017. doi: 10.1007/s11012-017-0749-3.

[33] C. Lee and F. C. Filippou. Frame elements with mixed formulation for singular section response. Int J Numer Meth Engng, 78:1320-1344, 2009. doi: 10.1002/nme.2531.

[34] F. B. Hildebrand. Introduction to numerical analysis, 2nd Ed. Dover Publications, Inc, New York, 1987.

[35] R. Luciano and E. Sacco. Variational methods for the homogenization of periodic heterogeneous media. Euro J Mech A-Solid, 17(4):599-617, 1998. doi: 10.1016/S09977538(99)80024-2. 
[36] E. Sacco. A nonlinear homogenization procedure for periodic masonry. European Journal of Mechanics - A/Solids, 28(2):209-222, 2009.

[37] A. Saritas and O. Soydas. Variational base and solution strategies for non-linear force-based beam finite elements. Int J Nonlinear Mech, 47(3):54-64, 2012. doi: 10.1016/j.ijnonlinmec.2012.01.003.

[38] M. H. Scott and G. L. Fenves. Plastic hinge integration methods for force-based beamcolumn elements. J Struct Eng - ASCE, 132(2):244-252, 2006. doi: 10.1061/(ASCE)07339445(2006)132:2(244).

[39] J. Donea and L. G. Lamain. A modified representation of transverse shear in C0 quadrilateral plate elements. Comput Method Appl M, 63(2):183-207, 1987.

[40] G. Prathap and C. R. Babu. An isoparametric quadratic thick curved beam element. Int J Numer Meth Eng, 23(9):1583-1600, 1986.

[41] I. Cancelliere, M. Imbimbo, and E. Sacco. Experimental tests and numerical modeling of reinforced masonry arches. Eng Struct, 32:776-792, 2010. doi: 10.1016/j.engstruct.2009.12.005.

[42] D. Addessi, E. Sacco, and P. Di Re. A micro-macro homogenization for modeling the masonry out-of-plane response. In XXIII Conference of The Italian Association of Theoretical and Applied Mechanics - AIMETA, September 2017.

[43] E. Bertolesi, G. Milani, and R. Fedele. Fast and reliable non-linear heterogeneous FE approach for the analysis of FRP-reinforced masonry arches. Compos Part B - Eng, 88(3): 189-200, 2016.

[44] D. V. Oliveira, I. Basilio, and P. B. LourenÃSo. Experimental Behavior of FRP Strengthened Masonry Arches. J Compos Constr, 14(3):312-322, 2010. doi: 10.1061/(ASCE)CC.19435614.0000086 .

[45] M. R. Valluzzi, M. Valdemarca, and C. Modena. Behavior of Brick Masonry Vaults Strengthened by FRP Laminates. J Compos Constr, 5(3):163-169, 2001. doi: 10.1061/(ASCE)10900268(2001)5:3(163).

[46] A. T. Vermeltfoort. Analysis and experiments of masonry arches. Historical Constructions, pages 489-498, 2001. 
[47] G. Milani and A. Tralli. A simple meso-macro model based on SQP for the non-linear analysis of masonry double curvature structures. Int J Solids Struct, 49(5):808-834, 2012. doi: 10.1016/j.ijsolstr.2011.12.001.

[48] A. Chiozzi, G. Milani, and A. Tralli. Fast kinematic limit analysis of FRP-reinforced masonry vaults II: numerical simulations. J Eng Mech, 143(9):04017072, 2017. doi: 10.1061/(ASCE)EM.1943-7889.0001268. 